Claremont Colleges

Scholarship@ Claremont

All HMC Faculty Publications and Research

HMC Faculty Scholarship

$1-1-1997$

\title{
The Interaction of a Point Vortex with a Wall- bounded Vortex Layer
}

Oliver V.Atassi

Northwestern University

Andrew J. Bernoff

Harvey Mudd College

Seth Lichter

Northwestern University

\section{Recommended Citation}

OLIVER V. ATASSI, ANDREW J. BERNOFF and SETH LICHTER (1997). The interaction of a point vortex with a wall-bounded vortex layer. Journal of Fluid Mechanics, 343, pp 169-195 doi:10.1017/S0022112097005867

This Article is brought to you for free and open access by the HMC Faculty Scholarship at Scholarship @ Claremont. It has been accepted for inclusion in All HMC Faculty Publications and Research by an authorized administrator of Scholarship @ Claremont. For more information, please contact scholarship@cuc.claremont.edu. 


\title{
The interaction of a point vortex with a wall-bounded vortex layer
}

\author{
By OLIVER V. ATASSI ${ }^{1}$, ANDREW J. BERNOFF \\ AND SETH LICHTER ${ }^{1}$ \\ ${ }^{1}$ Department of Mechanical Engineering, Northwestern University, Evanston, IL 60208, USA \\ ${ }^{2}$ Department of Engineering Sciences and Applied Mathematics, \\ Northwestern University, Evanston, IL 60208 USA
}

(Received 29 February 1996 and in revised form 14 February 1997)

The interaction of a point vortex with a layer of constant vorticity, bounded below by a wall and above by an irrotational flow, is investigated as a model of vortexboundary layer interaction. This model calculates both the evolution of the interface which separates the vortex layer from the irrotational flow and the trajectory of the vortex. In order to determine the conditions which lead to sustained unsteady interaction, three cases are investigated where the mutual interaction between the vortex and interface is initially assumed to be weak. (i) When a weak point vortex lies outside the layer, the vortex moves with a horizontal speed that is small relative to the long-wave phase speed of interfacial waves. A uniformly valid solution is found for the interface evolution. This solution shows that for long times the interface and the vortex approach an equilibrium state. (ii) When a weak vortex lies inside the layer, the vortex is convected by the mean flow and moves with a horizontal speed which matches the phase speed of an interfacial wave. This results in a strong interaction between the vortex and the interfacial wave. On the interface, a monochromatic wavetrain forms upstream of the vortex and acts to attract or repel the point vortex. The displacement of the vortex due to the wavetrain results in the modulation of the amplitude and wavelength of the wavetrain. If the point vortex is attracted toward the interface the horizontal speed of the vortex slows and disturbances directly above the vortex focus and grow leading to the ejection of vorticity. (iii) When the point vortex lies close to the wall and it is sufficiently strong it propagates downstream with a large horizontal velocity. In this case, the amplitude of the interfacial disturbance is independent of the vortex strength. Again, the vortex and the interface approach an equilibrium state. The results of this paper indicate that when the horizontal speed of the vortex matches the phase speed of the interfacial disturbance, it is necessary to account for the vertical displacement of the vortex in order to predict the behaviour of vortex-boundary layer interactions.

\section{Introduction}

For high-Reynolds-number flows the time scale associated with the evolution of the flow is often much smaller than the viscous time scale. Consequently, the effects of viscosity may be neglected or confined to thin boundary layers. The bulk of the flow may then be treated as inviscid but made up of vortical and irrotational regions. Wellknown examples of such flows are boundary layers, jets and wakes. Other examples include flows with trailing vortices or turbulent boundary layers with evolving patches 
of vorticity. Owing to the interaction between the various flow regions, such flows are almost always unsteady.

The method of contour dynamics, introduced by Zabusky, Hughes \& Roberts (1979), provides a means to investigate the evolution of inviscid flows with regions of vorticity. This method assumes a piecewise constant vorticity distribution and numerically calculates the Lagrangian evolution of the interfaces separating the various regions of vorticity. Contour dynamics has been extended by Dritschel (1988a) to enable the long-time simulation of regions of uniform vorticity and the cascade to fine scales. It is a computationally efficient means for studying inviscid flows because it maps the two-dimensional Euler equations to a one-dimensional Lagrangian formulation along the contours. It has also been used to study boundary layer flows (Pullin 1981; Stern \& Pratt 1985; Jimenez \& Orlandi 1993), where it was found that small-amplitude disturbances propagate as dispersive waves and that finite-amplitude disturbances exhibit nonlinear steepening and overturning that result in the ejection of thin filaments of vorticity and the entrainment of irrotational flow.

Models involving the interaction of a point vortex with an interface separating two regions of constant vorticity have been studied for geophysical applications (Stern \& Flierl 1987; Bell 1990). For such geometries (in contrast to the wall-bounded layer studied here) the interfacial (linear) waves are non-dispersive. These studies showed that when the point vortex moves with a speed that coincides with the phase speed of the interfacial waves a resonant interaction occurs resulting in a sinusoidal wavetrain upstream of the point vortex. The effect of this wavetrain acts to attract (repel) the vortex toward (away from) the interface.

In this paper, we study the mutual interaction of a point vortex with a wallbounded layer of constant vorticity. This model problem is motivated by observations that boundary layer interactions with localized patches of vorticity play a major role in bursting and ejection phenomena (Doligalski, Smith \& Walker 1994). The paper aims at determining the physical processes underlying vortex-boundary layer interaction which are important for characterizing the conditions under which the transition from a quasi-steady laminar flow to a highly unsteady flow occurs.

Boundary layer theory has been used to investigate vortex-boundary layer interactions (Walker 1978; Peridier, Smith \& Walker 1991). It has been demonstrated that the process of boundary layer ejection can be initiated by a local concentration of vorticity which then results in a rapidly rising filament of boundary layer fluid that ultimately interacts strongly with the external flow. In addition, these models have verified the hypothesis (Sears \& Telionis 1975) that a finite-time singularity occurs in the boundary layer equations when the interaction between the point vortex and the boundary layer becomes strong (Van Dommelen \& Shen 1980; Peridier et al. 1991).

The contour dynamics model we are presenting is computationally efficient for high-Reynolds-number flows and circumvents the difficulty of the breakdown of the boundary layer equations when the interaction between the point vortex and the layer become strong. The present model does not break down when the vortex layer is ejected away from the wall and the phenomena of wave breaking and rollup that result in multi-valued deformations of the contour pose no difficulty. However, the model does not account for the vorticity production that occurs over time near the wall. Consequently, the results presented here are useful at high Reynolds number, when the boundary is being deformed quickly in comparison with the viscous time scale; otherwise, the effects of vorticity produced at the wall may overwhelm the effects of the point vortex. Section 6 discusses this criterion.

The aims of this work are to (i) describe the mutual interaction of the point 
vortex and the vortex layer in terms of the evolution of the interface and the trajectory of the point vortex and (ii) identify the various physical processes involved in the interaction between a point vortex and a wall-bounded vortex layer. We are particularly interested in the conditions under which a transition to sustained unsteady flow occurs as opposed to a transient period of unsteady interaction. In general, this problem is nonlinear and must be solved numerically. However, for certain asymptotic cases linear theories are developed and analytical solutions obtained. These provide insight into the physical processes and may describe the preliminary stages of the onset of nonlinear behaviour. It should be pointed out that in certain cases our linearized solutions account for the effect of the layer on the motion of the vortex, which could be essential for determining the long-term behaviour of the system and its transition from linear to nonlinear behaviour. In particular, we find that for a weak vortex placed inside the layer the vertical displacement of the vortex acts to modulate the wavelength and amplitude of the wavetrain. When the vortex is attracted to the interface, steepening and growth of disturbances directly above the vortex occurs.

The problem depends on two parameters: the vortex strength, $\tilde{\Gamma}$, and the initial $y$-coordinate, $Y(0)$, of the point vortex (see §3.1). These two parameters indicate the strength of the mutual interaction between the point vortex and the vortex layer and thus determine the type of behaviour seen, such as a dispersive wave, modulated wavetrain or ejection. The horizontal speed of the vortex, $c_{v}$, is often a useful indicator of the type of linear interaction that will occur. As we will see, there is a range of speeds for which a resonant interaction between the point vortex and the vortex layer is possible. For vortices translating much slower than those which are resonant, dispersive waves may occur; for vortices which are much faster, steady compact waves of non-changing form are possible.

In $\S 2$, we present the formulation of our model. Section 3 presents the linear analysis for three cases. For a weak point vortex lying above a vortex layer, a uniformly valid asymptotic solution is found which consists of a travelling wave plus a transient which disperses. In the case where the vortex is weak and lies within the vortex layer, it is mainly convected by the unperturbed flow, $c_{v}=O(1)$. Thus, when $0<c_{v}<1$, a resonant interaction occurs between the point vortex and that interfacial wave whose phase speed coincides with the vortex speed. Finally, in $\$ 3.5$ we consider the case of a point vortex placed very close to the wall and whose strength is such that the speed of the point vortex is much larger than the phase speed of the interfacial waves, $c_{v} \gg 1$. In this case, a non-dispersive wave is formed which propagates downstream in tandem with the point vortex and leaves dispersive waves far behind it. The amplitude of these disturbances is found to be independent of the strength of the vortex. In $\S 4$, we use conservation of $x$-momentum to obtain bounds on the vertical displacement of the vortex in terms of the norm of the interfacial displacement. The vortex acts to transfer momentum to the interface, while the interface then both transfers the momentum back to the vortex, thereby drawing the vortex towards or away from the interface, and carries it downstream via dispersive waves (see Bell 1990). In §5, we present numerical results for the cases studied analytically in $\S 3$ and for the transition to weakly and strongly nonlinear behaviour where these analytical solutions break down. In §6, we summarize our work and present our conclusions.

\section{Formulation}

We consider a two-dimensional inviscid incompressible flow bounded by a wall, see figure 1 . The flow is divided into two regions: an inner region $D_{+}$of constant vorticity 


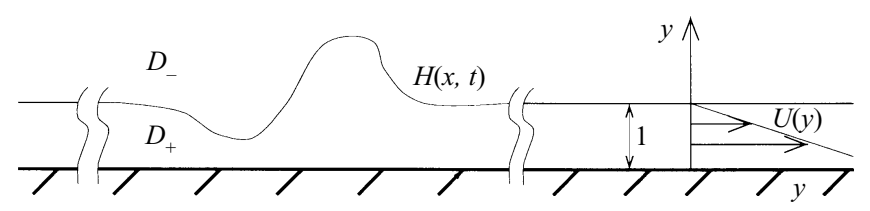

FIGURE 1. A schematic of the geometry in dimensionless variables in a coordinate system moving with the uniform free-stream velocity. A vortex of circulation $\tilde{\Gamma}$ is located a distance $Y(t)$ above the wall. The vortex is initially located at $Y(0)>H_{0}(x)$ but it can also be placed within the vortex layer $Y(0)<H_{0}(x)$. In this coordinate system, the wall is moving to the right and the free-stream velocity is zero. As the flow field evolves with time, the vortical fluid is contained within the region $D_{+}$which is bounded by a curve $C$ consisting of the interface $y=H(x, t)$, the wall $y=0$ and closed at $x= \pm \infty$. The region $D_{-}$contains irrotational flow.

$\omega$ adjacent to the wall, and an outer region $D_{-}$of irrotational flow extending to infinity with a free-stream velocity $U$. The two regions are separated by the interface located at $y=H(x, t)$. The vortex layer extends upstream to $-\infty$ and downstream to $+\infty$ where it has constant thickness $H_{\infty}$, and thus $\omega=U / H_{\infty}$.

We consider a frame of reference moving with the free-stream velocity $U$, and a coordinate system $(x, y)$ centred at the wall with the $x$-axis parallel to the wall and the $y$-axis perpendicular to it. We non-dimensionalize all lengths with respect to $H_{\infty}$, and time with respect to $H_{\infty} / U$. In this frame of reference the fluid velocity vanishes as $y \rightarrow \infty$ and the wall, located at $y=0$, appears to be moving to the right.

Using a complex representation, the complex conjugate of the velocity induced by the vortex layer is given by

$$
u-\mathrm{i} v=\frac{1}{2 \pi \mathrm{i}} \iint_{D_{+}}\left[\frac{1}{z-z^{\prime}}-\frac{1}{z-\bar{z}^{\prime}}\right] \mathrm{d} A^{\prime},
$$

where $z^{\prime}$ represents the source point and $z$ the observation point. We apply the area theorem (Milne-Thomson 1968, p. 133) to transform the double integral over the area $D_{+}$to a line integral over the contour C enclosing the region $D_{+}$(see also Pullin 1981). This yields

$$
u-\mathrm{i} v=\frac{1}{2 \pi \mathrm{i}}\left[\oint_{C} \frac{y^{\prime}-y}{z^{\prime}-z} \mathrm{~d} z^{\prime}-\oint_{C} \frac{y^{\prime}-y}{\bar{z}^{\prime}-z} \mathrm{~d} \bar{z}^{\prime}\right],
$$

where the direction of integration is chosen to be counterclockwise. Note that the assumption that the layer is of finite thickness 1 as $|x| \rightarrow \infty$ ensures that the velocity field is finite. The expression for the velocity (2.2) is particularly suitable for accurate numerical quadrature because it no longer involves a singular kernel. Moreover, these integrals can be evaluated analytically for a vortex layer of constant thickness. Thus the numerical quadrature can be limited to the perturbed part of the contour, resulting in a significant saving of computation time.

Any potential field which satisfies the boundary conditions can be added to (2.2). In particular, we are interested in the mutual interaction of the vortex layer and a point vortex of circulation $\tilde{\Gamma}$, as illustrated in figure 1 . If the position of the point 
vortex is $(X(t), Y(t))$ then the total velocity is given by

$$
u-\mathrm{i} v=\frac{1}{2 \pi \mathrm{i}}\left[\oint_{C} \frac{y^{\prime}-y}{z^{\prime}-z} \mathrm{~d} z^{\prime}-\oint_{C} \frac{y^{\prime}-y}{\bar{z}^{\prime}-z} \mathrm{~d} \bar{z}^{\prime}\right]+\frac{\tilde{\Gamma}}{2 \pi \mathrm{i}}\left(\frac{1}{z-Z}-\frac{1}{z-\bar{Z}}\right),
$$

where $Z=X(t)+\mathrm{i} Y(t)$ and the non-dimensional parameter $\tilde{\Gamma}=\Gamma /\left(\omega H_{\infty}^{2}\right)$ represents the ratio of the strength of the point vortex non-dimensionalized with respect to the vorticity and unperturbed layer thickness. We further note that (2.3) can be written as

$$
\begin{aligned}
u-\mathrm{i} v=\frac{1}{2 \pi \mathrm{i}} \oint_{C} \frac{\left(y^{\prime}-1\right)}{z^{\prime}-z} \mathrm{~d} z^{\prime} & -\oint_{C} \frac{\left(y^{\prime}-1\right)}{\bar{z}^{\prime}-z} \mathrm{~d} \bar{z}^{\prime} \\
& +\left\{\begin{array}{ll}
1-y & \text { if } z \in D_{+} \\
0 & \text { if } z \in D_{-}
\end{array}+\frac{\tilde{\Gamma}}{2 \pi \mathrm{i}}\left(\frac{1}{z-Z}-\frac{1}{z-\bar{Z}}\right) .\right.
\end{aligned}
$$

The motion of the point vortex depends upon its interaction with the wall and the vortex layer, thus its complex-conjugate velocity is given by

$$
X_{t}-\mathrm{i} Y_{t}=\frac{\mathrm{d} \bar{Z}}{\mathrm{~d} t}=\frac{1}{2 \pi \mathrm{i}}\left[\oint_{C} \frac{y^{\prime}-Y}{z^{\prime}-Z} \mathrm{~d} z^{\prime}-\oint_{C} \frac{y^{\prime}-Y}{\bar{z}^{\prime}-Z} \mathrm{~d} \bar{z}^{\prime}\right]+\frac{\tilde{\Gamma}}{4 \pi Y} .
$$

Finally, we must specify the initial shape of the interface, $y=H(x, 0)=H_{0}(x)$ and the kinematic condition $\dagger$ that a fluid particle on the interface will remain on the interface,

$$
v=\frac{\partial H}{\partial t}+u \frac{\partial H}{\partial x} \quad \text { at } \quad y=H(x, t) .
$$

The problem of mutual interaction between a wall-bounded vortex layer and a point vortex is thus formulated in terms of an initial value problem involving three coupled equations (2.3), (2.5), (2.6). This initial value problem defining the coupled motions of the interface and the point vortex is nonlinear, in general, and must be solved numerically. However, since a variety of phenomena such as dispersion, steepening, rollup and ejection of the vortex layer have been observed numerically, it is useful to determine analytical solutions for asymptotic cases to interpret the physics underlying these various phenomena. With this in mind, before presenting the results of the fully nonlinear simulations, we first investigate, in $\S 3$, some limiting cases where the equations of motion are linear and so are soluble analytically.

\section{The linear interaction of a vortex layer with a point vortex}

In this section, we assume that the interface undergoes only small departures from its initially unperturbed position $(y=1)$ and that the mutual interaction between the vortex and the interface is weak. These restrictions allow us to linearize the problem and obtain analytical results. First, we will linearize the velocity fields (2.3), (2.5) and the interface condition (2.6) to derive the dispersion relation. Then we consider two cases where the vortex is weak $(\tilde{\Gamma} \ll 1)$ and where the forcing lies either outside $(Y>1)$ or inside $(Y<1)$ the vortex layer. Finally, we will consider the case where the vortex is placed close to the wall and propagates downstream with a large horizontal velocity, $c_{v} \gg 1$ (those vortices whose speed $c_{v} \gg 1\left(c_{v} \ll 1\right)$ will often be referred to as

$\dagger$ Note that (2.6) will have a different form when the interface becomes multi-valued. In this case one can parameterize $x=x(s, t)$ and $y=y(s, t)$ as functions of some parameter $s$, for example arclength, such that $x$ and $y$ are single-valued functions. 
'fast' ('slow')). In this case, the interaction of the vortex with the wall dominates the motion of the vortex. Unlike the previous two cases, the linearized velocity field in (3.5) does not apply since the velocity induced by the vortex on the interface is large. However, because the horizontal vortex speed is so great, the effect of the vortex is felt for only a fleeting interval, leaving small disturbances on the interface. In this case, the equations describing the velocity field need to be rescaled as is shown in $§ 3.5$.

\subsection{The linearized velocity field and kinematic condition}

We consider the interaction of a vortex layer with a weak point vortex initially located at $(0, Y(0))$ where it may be placed above the interface $(Y(0)>1)$ or inside the vortex layer $(Y(0)<1)$. We linearize about an initially flat interface,

$$
H(x, t)=1+\epsilon \eta(x, t),
$$

where the parameter, $0<\epsilon \ll 1$. Furthermore, we assume $\tilde{\Gamma}=\epsilon \Gamma^{*}$, with $\left|\Gamma^{*}\right|=O(1)$. We expand both the velocity field and the coordinate location of the point vortex as

$$
u-\mathrm{i} v=(u-\mathrm{i} v)_{0}+\epsilon(u-\mathrm{i} v)_{1}+\cdots
$$

and

$$
(X(t), Y(t))=\left(X_{0}(t), Y_{0}(t)\right)+\epsilon\left(X_{1}(t), Y_{1}(t)\right)+\cdots .
$$

Substituting (3.1-3.3) into (2.4) we obtain

$$
(u-\mathrm{i} v)_{0}= \begin{cases}1-y & \text { if } z \in D_{+} \\ 0 & \text { if } z \in D_{-}\end{cases}
$$

and

$$
\begin{aligned}
(u-\mathrm{i} v)_{1}= & -\frac{1}{2 \pi \mathrm{i}} \int_{-\infty}^{\infty} \frac{\eta\left(x^{\prime}, t\right)}{\left(x^{\prime}-x\right)+\mathrm{i}(1-y)} \mathrm{d} x^{\prime}+\frac{1}{2 \pi \mathrm{i}} \int_{-\infty}^{\infty} \frac{\eta\left(x^{\prime}, t\right)}{\left(x^{\prime}-x\right)-\mathrm{i}(1+y)} \mathrm{d} x^{\prime} \\
& +\frac{\Gamma^{*}}{2 \pi \mathrm{i}}\left[\frac{1}{\left(x-X_{0}(t)\right)+\mathrm{i}\left(y-Y_{0}(t)\right)}-\frac{1}{\left(x-X_{0}(t)\right)+\mathrm{i}\left(y+Y_{0}(t)\right)}\right] .
\end{aligned}
$$

We introduce the shorthand $Y_{0}(0)=d$ where we assume that $d=O(1)$ and $|d-1|=$ $O(1)$. To leading order, the vertical velocity of the vortex is zero and its horizontal velocity, $c_{v}$, is constant,

$$
c_{v}= \begin{cases}0 & \text { if } d>1 \\ 1-d & \text { if } d<1\end{cases}
$$

As a result, $X_{0}(t)=c_{v} t$ and $Y_{0}(t)=d$ and therefore, the velocity field induced by the vortex is independent of the evolution of the interface. Note that (3.6) is not uniformly valid as $t$ becomes large. The range of time for which it is valid is discussed in $\$ 3.3 .3$.

The linearized kinematic condition reduces to

$$
\eta_{t}=v_{1} .
$$

Thus, to first order, only the vertical component of the velocity contributes to the evolution of the interface. The vertical velocity at the interface, $v_{1}$, is obtained from (3.5) by taking $y=1$ and treating the first integral as a Cauchy principal value. Substituting the expression for $v_{1}$ into (3.7) and taking the Fourier transform

$$
\mathscr{F}\{\eta(x, t)\}=\hat{\eta}(k, t)=\frac{1}{2 \pi} \int_{-\infty}^{\infty} \eta(x, t) \mathrm{e}^{-\mathrm{i} k x} \mathrm{~d} x,
$$




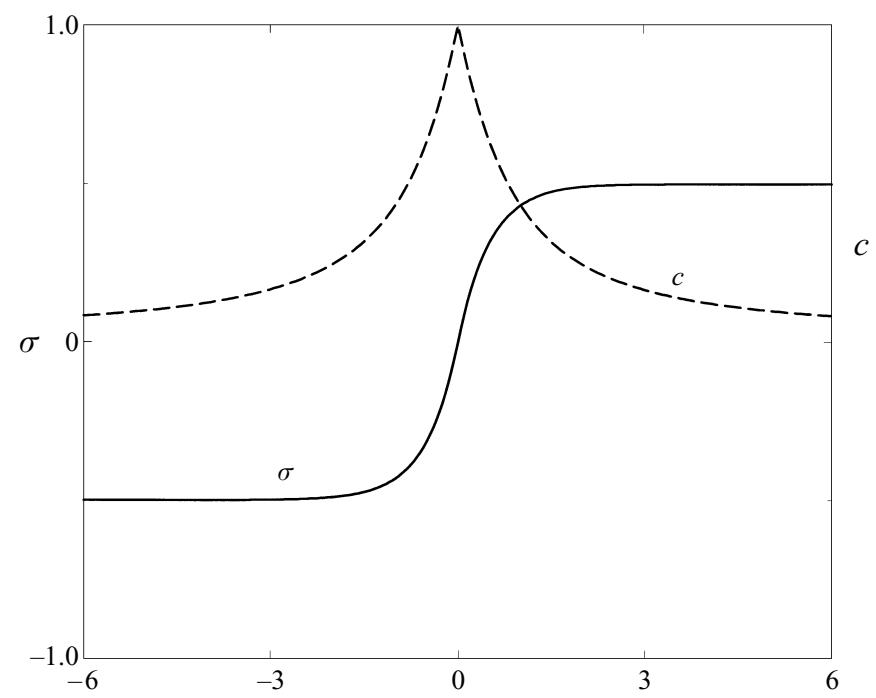

FIGURE 2. A plot of the dispersion relation and the phase velocity where $\sigma$ is the angular frequency, $c$ is the phase velocity and $k$ is the wavenumber. Note that the frequency is antisymmetric with respect to $k$ and the phase velocity is symmetric with respect to $k$.

we find

$$
\frac{\mathrm{d} \hat{\eta}}{\mathrm{d} t}=-\mathrm{e}^{-|k|} \sinh (k) \hat{\eta}-\mathrm{i} \frac{\Gamma^{*}}{2 \pi} \begin{cases}\mathrm{e}^{-d|k|} \sinh (k) \mathrm{e}^{-\mathrm{i} k c_{v} t} & \text { if } d>1 \\ \mathrm{e}^{-|k|} \sinh (k d) \mathrm{e}^{-\mathrm{i} k c_{v} t} & \text { if } d<1 .\end{cases}
$$

\subsection{Dispersion relation}

Since the point-vortex forcing is independent of the evolution of the interface we can obtain the dispersion relation for this problem by examining sinusoidal disturbances to the interface in the absence of forcing. This dispersion relation is readily obtained by setting $\tilde{\Gamma}=0$ in (3.9) and substituting solutions of the form $\hat{\eta}(k, t)=\tilde{\eta}(k) \mathrm{e}^{-\mathrm{i} \sigma t}$, where $\sigma$ is the angular frequency. This yields the well-known (Rayleigh 1887; Pullin 1981; Stern \& Pratt 1985) dispersion relation,

$$
\sigma(k)=\mathrm{e}^{-|k|} \sinh (k)
$$

shown in figure 2 .

From (3.10) we find the phase velocity

$$
c=\mathrm{e}^{-|k|} \frac{\sinh (k)}{k}
$$

and the group velocity

$$
c_{g}=\mathrm{e}^{-2|k|}
$$

In the long-wave limit the phase velocity reduces to

$$
c=1-|k|+\cdots
$$

and in the short-wave limit

$$
c=\frac{1}{2|k|} .
$$

As a result, linear theory indicates that small disturbances to the interface will 
propagate as dispersive waves where disturbances of longer wavelength will propagate faster than disturbances of short wavelength. Note that the dispersive nature of the wave is caused by the presence of the wall; without the wall the frequency would be constant, $\sigma=1 / 2$.

\subsection{Weak vortex outside the layer}

We consider the response of the vortex layer to a weak point vortex initially located at $(0, d)$ which is outside $(d>1)$ the vortex layer. In this limit, $c_{v}=0$ and for $t$ not too large, (3.9) becomes

$$
\frac{\mathrm{d} \hat{\eta}}{\mathrm{d} t}=-\mathrm{i}\left[\mathrm{e}^{-|k|} \hat{\eta}+\frac{\Gamma^{*}}{2 \pi} \mathrm{e}^{-d|k|}\right] \sinh (k) .
$$

This has the solution

$$
\hat{\eta}=c_{1}(k) \mathrm{e}^{-\mathrm{i}\left[\sinh (k) \mathrm{e}^{-k k}\right] t}-\frac{\Gamma^{*}}{2 \pi} \mathrm{e}^{-(d-1)|k|} .
$$

The first term is the homogeneous solution to (3.9) and corresponds to a dispersive wave that is propagating downstream due solely to the effect of the vortex layer. The second term corresponds to the particular solution and is a time-independent solution that results from the forcing of the point vortex. Thus, the effect of the point vortex is to form a steady solution directly below it, while the effect of the vortex layer is to create dispersive waves on the interface. The relative magnitude of these two effects is measured by the non-dimensional parameter $\Gamma^{*}$.

\subsubsection{Interface response}

The interface response is obtained by finding the inverse Fourier transform of $\hat{\eta}$ in (3.16). To this end, we first note that the steady part of (3.9) yields

$$
\bar{S}(x)=-\frac{\Gamma^{*}}{\pi}\left[\frac{(d-1)}{(d-1)^{2}+x^{2}}\right] .
$$

The form of this solution indicates that a vortex of positive (negative) vorticity will create a steady, trough (hump) at the interface. To complete the initial value problem we apply the initial condition, $\eta_{0}=\eta(x, 0)$, whose Fourier transform is $\hat{\eta}(k, 0)$, to determine $c_{1}(k)$ in (3.16). This gives

$$
c_{1}(k)=\hat{\eta}_{0}+\frac{\Gamma^{*}}{2 \pi} \mathrm{e}^{-|k|(d-1)} .
$$

From this, the full linear response of the vortex layer to the point vortex can be written in terms of $\hat{\eta}_{0}$ and yields

$$
\eta(x, t)=\int_{-\infty}^{\infty} \hat{\eta}_{0} \mathrm{e}^{\mathrm{i} k(x-c t)} \mathrm{d} k+\int_{-\infty}^{\infty} \frac{\Gamma^{*}}{2 \pi} \mathrm{e}^{-|k|(d-1)} \mathrm{e}^{\mathrm{i} k(x-c t)} \mathrm{d} k+\bar{S}(x),
$$

where we have assumed that $\hat{\eta}_{0}$ decays for high wavenumbers so that the first integral converges. If we examine (3.19), we find that it is the sum of three contributions. One is the steady trough (hump) solution which represents the long-time effect of the vortex forcing. Another is a time-dependent solution which initially cancels the steady solution but then propagates away dispersively. The third contribution is due to the initial shape of the interface. 


\subsubsection{The long-time behaviour of the interface}

To determine the long-time evolution of the system we examine the evolution of the interface for $t$ large. We first examine the behaviour of the time-dependent part of the solution (3.19) due to the vortex forcing. This is equivalent to the interface being initially flat, $\hat{\eta}_{0}=0$. For large $t$, there are three leading-order contributions to the integrals in (3.19). Two come from $k \rightarrow 0$ and $k \rightarrow \infty$, which correspond to the long- and short-wave contributions. The third contribution comes from the point of stationary phase, where $k=\tilde{k}$ such that $c_{g}=x / t$. Note that when $x=O(1)$, $c_{g} \rightarrow 0$ and $\tilde{k} \rightarrow \infty$. Thus the contribution of the stationary phase point is the same as that of the short-wave endpoint. On the other hand, for large $x$ such that $x=O(t)$, $\tilde{k}=-\frac{1}{2} \ln (x / t)$ which is finite.

For large $t$, the interface response in (3.19) can be written in terms of a gamma function (the details of which are given in the Appendix). Equations (A 6) and (A 7) show that the long-time contribution to the interface disturbance comes mainly from the short- and long-wave limits. If the vortex is not too far from the interface $(1<d<5)$ the leading contribution to the disturbance comes from short waves of $O\left(1 / t^{(d-1) / 2}\right)$ as is shown in the expression

$$
\begin{aligned}
\eta(x, t) \sim \frac{\Gamma^{*}}{2 \pi}\left[\frac{2^{(d-1) / 2}}{t^{(d-1) / 2}} \mathrm{e}^{\pi x / 4} \operatorname{Re}\{\right. & \operatorname{exp~\mathrm {i}}\left[\frac{-t}{2}+\frac{x}{2} \ln \left(\frac{t}{2}\right)\right. \\
& \left.\left.\left.+\frac{\pi}{4}(d-1)\right] \Gamma\left(\frac{(d-1-\mathrm{i} x)}{2}\right)\right\}\right]+\bar{S}(x),
\end{aligned}
$$

while the long-wave contribution is $O\left(1 / t^{2}\right)$. On the other hand, when the vortex is located far from the interface $(d>5)$ the long-term contribution is mainly due to the long waves and decays like $1 / t^{2}$.

The dependence of the rate of decay of the transient short-wave solution (3.20) on the distance of the point vortex above the interface indicates the importance of the interaction of the point vortex with the short waves on the interface. Thus when the point vortex is initially placed close to the interface, some short-wave contributions will subsist for long times and will appear as standing waves with a frequency close to $1 / 2$ and it is these short waves which will interact strongly with the vortex.

Asymptotic solutions can be obtained for both small and large $x$. Expanding the complex Gamma function for small argument (see Gradshteyn \& Ryzhik 1994), i.e. $\left((d-3)^{2}+x^{2}\right)<4$, we find

$$
\eta(x, t) \sim \frac{\Gamma^{*}}{2 \pi} \frac{2^{(d-1) / 2}}{t^{(d-1) / 2}} \cos \left[\frac{t}{2}-\left(\frac{\pi(d-1)}{4}+\frac{x}{2} \ln \left(\frac{t}{2}\right)\right)\right]+\bar{S}(x) .
$$

This solution indicates that for the region of the interface just below the vortex a nearly stationary decaying oscillation with a frequency of $1 / 2$ takes place. Thus we can conclude that for very long times only the steady solution, $\bar{S}(x)$, will remain.

For large $x=O(t)$, we find

$$
\eta(x, t) \sim \frac{\Gamma^{*}}{2 \pi^{1 / 2}} \frac{(x / t)^{(d-1) / 2} \cos \left[\frac{1}{2}(t-x)+\frac{1}{2} x \ln (x / t)-\frac{1}{4} \pi\right]}{x^{1 / 2}} .
$$

This solution indicates that far downstream of the origin very long waves will decay like $1 / x^{1 / 2}$

We now consider the behaviour of the time-dependent solution resulting from an initial disturbance to the interface specified by $\eta_{0}(k)$. We assume that $\hat{\eta}_{0}$ decays 
exponentially as $|k| \rightarrow \infty$, i.e. $\hat{\eta}_{0}=\mathrm{e}^{-\gamma|k|} / \gamma$ where $\gamma>0$, corresponding to an initial distribution of the form $\eta_{0}=1 /\left(x^{2}+\gamma^{2}\right)$. In this case, using (A 7) we see that

$$
\eta(x, t) \sim \frac{\mathrm{e}^{-\mathrm{i} t / 2}}{t^{\gamma / 2}} .
$$

This example illustrates the possibility that the long-term behaviour of the transient solution could be dominated either by the form of the initial disturbance to the interface or by the presence of the vortex depending upon the value of $\gamma$ and $d$ as can be seen by comparing (3.20) and (3.23). In all cases the transient solution will have an asymptotic frequency of $1 / 2$ corresponding to the limit as the wavelength of the disturbance vanishes. This result is not surprising since we are dealing with a dispersive system where the long waves propagate faster than the short waves.

The aforementioned analysis of the interface motion suggests that the slow timedependent motion of the point vortex may also exhibit a similar decaying oscillatory behaviour with a frequency of $1 / 2$. In addition, for long times the vortex will have drifted an order one distance and thus must be accounted for in the solution. In the next section, we examine the large-time motion of the vortex and extend the validity of the solution to all $t$.

\subsubsection{The motion of the point vortex}

In this section, we determine the motion of the point vortex for long times. The velocity of the point vortex can be obtained from (3.5) and (3.6) and is expressed as

$$
X_{1_{t}}-\mathrm{i} Y_{1_{t}}=\frac{1}{2 \pi \mathrm{i}}\left\{-\int_{-\infty}^{\infty} \frac{\eta\left(x^{\prime}, t\right)}{x^{\prime}-\mathrm{i}(d-1)} \mathrm{d} x^{\prime}+\int_{-\infty}^{\infty} \frac{\eta\left(x^{\prime}, t\right)}{x^{\prime}-\mathrm{i}(d+1)} \mathrm{d} x^{\prime}\right\}+\frac{\Gamma^{*}}{4 \pi d}+O\left(\epsilon^{2}\right) .
$$

Substituting the time-dependent part of the solution of (3.19) with $\hat{\eta}_{0}(k, t)=0$ into (3.24) and applying the residue theorem, we obtain

$$
X_{1_{t}}^{(u)}=-\frac{\Gamma^{*}}{\pi} \int_{0}^{\infty} \mathrm{e}^{-k(2 d-1)} \sinh (k) \cos (\sigma t) \mathrm{d} k
$$

and

$$
Y_{1_{t}}^{(u)}=-\frac{\Gamma^{*}}{\pi} \int_{0}^{\infty} \mathrm{e}^{-k(2 d-1)} \sinh (k) \sin (\sigma t) \mathrm{d} k,
$$

where the superscript $(u)$ denotes the unsteady contribution. Since the integrand vanishes for small and large $k$, the contribution of the interfacial waves to the vortex velocity comes mainly from wavelengths in the mid-range. The distance travelled by the vortex due to the transient can be obtained by integrating (3.25) and (3.26) with respect to $t$,

$$
X_{1}^{(u)}=-\frac{\Gamma^{*}}{\pi} \operatorname{Im}\left\{\mathrm{e}^{-\mathrm{i} t / 2} J(d-2, t)\right\}
$$

and

$$
Y_{1}^{(u)}=-\frac{\Gamma^{*}}{2 \pi(d-1)}+\frac{\Gamma^{*}}{\pi} \operatorname{Re}\left\{\mathrm{e}^{-i t / 2} J(d-2, t)\right\},
$$

where we have used the dispersion relation given by (3.10) to put the integrals into a form such that the results of the Appendix can be applied. The expression for $J(d-2, t)$ is given by (A 7) in the Appendix. For $1<d<2$ and large $t$ the leading-order terms 
of $J(d-2, t)$ are of the form

$$
X_{1}^{(u)}=-\frac{\Gamma^{*}}{2 \pi} \frac{2^{(d-1)} \Gamma(d-1)}{t^{(d-1)}} \sin [(t-\pi(d-1)) / 2]+O(\epsilon / t)
$$

and

$$
Y_{1}^{(u)}=-\frac{\Gamma^{*}}{2 \pi(d-1)}+\frac{\Gamma^{*}}{2 \pi} \frac{2^{(d-1)} \Gamma(d-1)}{t^{(d-1)}} \cos [(t-\pi(d-1)) / 2]+O(\epsilon / t),
$$

where in the above expressions the Gamma function $\Gamma$ should not be confused with the strength of the point vortex $\Gamma^{*}$. From the results of the previous section, we would expect that the decay of the transient would be slower when the point vortex is initially placed closer to the layer, and indeed this is the case. Note also that the contribution to the horizontal position of the point vortex vanishes with time leaving no net displacement while the vertical displacement of the vortex tends to a constant value.

These solutions corroborate the findings in the previous section that when the point vortex is initially placed close to the layer, the short-wave interaction between the vortex and the interface is stronger. Moreover, the time-dependent part of the vortex motion decays like $O\left(1 / t^{(d-1)}\right)$ which is faster than the decay of the time-dependent part of the interface solution, see (3.20).

We now account for the effect of the steady component of the vortex velocity $\left(X_{1_{t}}^{(s)}, Y_{1_{t}}^{(s)}\right)$. Examining (3.24) we see that one contribution comes from the term $\epsilon \Gamma^{*} /(4 \pi d)$ and that the second contribution, $\bar{S}(x)$, enters through the steady part of $\eta$. Since the steady part of $\eta$ is an even function, it induces a velocity only in the horizontal direction. As a result, the displacement of the vortex is

$$
\begin{gathered}
X_{1}^{(s)}=\frac{\epsilon \Gamma^{*}}{4 \pi(d-1)} t, \\
Y_{1}^{(s)}=0 .
\end{gathered}
$$

Finally, then, considering both transient and steady contributions to the displacement from the initial condition $(0, d)$, we find

$$
X(t)=c_{v_{\Gamma}} t+O(\epsilon / t)
$$

and

$$
Y(t)=d-\frac{\epsilon \Gamma^{*}}{2 \pi(d-1)}+O(\epsilon / t)
$$

where $c_{v_{\Gamma}}=\epsilon \Gamma^{*} /(4 \pi(d-1))$. This shows that because the effect of the time-dependent part of the interface decays with time, the point vortex will move with a uniform horizontal velocity due solely to the image vortex and the steady part of the solution, $\bar{S}(x)$. The vortex will also move from its original height to that given in (3.34) and then weakly oscillate about this value. Moreover, (3.33) and (3.34) show that the expansion for the vortex position is no longer valid for $t=O(1 / \epsilon)$. This suggests an outer expansion is needed for large $t$. Introducing the time, $T=\epsilon t$ and $C_{v_{\Gamma}}=c_{v_{\Gamma}} / \epsilon$, the last expression in (3.5) becomes

$$
\frac{\Gamma^{*}}{2 \pi \mathrm{i}}\left[\frac{1}{\left(x-C_{v_{\Gamma}} T\right)+\mathrm{i}(y-d)}-\frac{1}{\left(x-C_{v_{\Gamma}} T\right)-\mathrm{i}(y+d)}\right] .
$$


Repeating the analysis in $\S \S 3.1$ and 3.3 yields the outer solution which accounts for the vortex motion,

$$
S(x, T)=-\frac{\epsilon \Gamma^{*}}{\pi} \frac{(d-1)}{(d-1)^{2}+\left(x-C_{v_{\Gamma}} T\right)^{2}} .
$$

These results show that small-amplitude disturbances will not become large owing to the long-time drift of the point vortex towards the layer and that the vortex will approach an equilibrium state for large times. Moreover, as the effect of the transient decays, the interface will asymptotically evolve to a steady state in a reference frame moving with the vortex. Thus, a uniformly valid solution for any $t$ can be derived:

$$
\eta(x, t, T)=\int_{-\infty}^{\infty}\left\{\hat{\eta}_{0}+\frac{\Gamma^{*}}{2 \pi} \mathrm{e}^{-|k|(d-1)}\right\} \mathrm{e}^{\mathrm{i} k(x-c t)} \mathrm{d} k+S(x, T) .
$$

\subsection{Weak vortex inside the layer}

Here we investigate the linear response of the interface to a weak point vortex located at $(0, d)$ which is within $(d<1)$ the vortex layer. In this case, the point vortex is being convected by the vortex layer at a speed which is $O(1)$ while the velocity induced upon it by the perturbed vortex layer is $O(\epsilon)$. This also suggests that for $t$ not too large, the vertical motion of the vortex is $O(\epsilon)$, and thus the vortex is convected at a constant height, $c_{v} \sim 1-d$. Recall that the phase speed is $0<c(k)<1$. So, when the velocity of the point vortex is also $0<c_{v}<1$, there is a Fourier mode with wavenumber $\tilde{k}$ travelling at the same speed as the point vortex, $c(\tilde{k})=c_{v}$. This mode will be excited, resulting in a strong resonant interaction. Similar analyses carried out by Stern \& Flierl (1987) and Bell (1990) without the presence of a wall found that a spreading monochromatic wavetrain forms upstream of the vortex, and that this wave attracts the vortex toward or repels the vortex away from the interface depending upon whether $\tilde{\Gamma}$ is positive or negative.

In order to avoid the secular behaviour associated with resonance, we introduce damping in our system. Thus (3.9) becomes

$$
\frac{\mathrm{d} \hat{\eta}}{\mathrm{d} t}+\left[\mathrm{ie}^{-|k|} \sinh (k)+\alpha\right] \hat{\eta}=-\mathrm{i} \frac{\Gamma^{*}}{2 \pi} \mathrm{e}^{-\mathrm{i} k c_{v} t} \mathrm{e}^{-|k|} \sinh (k d),
$$

where $\alpha>0$ is a small damping parameter which adds dissipation to the system. Our solution will then be taken as the limit of the damped case where the dissipation coefficient, $\alpha$, approaches zero. This technique (see Stakgold 1967, pp. 259-261) is referred to as the principle of limiting absorption.

The transient homogeneous solution to (3.38) is

$$
\eta_{H}(x, t)=2 \operatorname{Re}\left\{\int_{0}^{\infty}\left[\hat{\eta}_{0}-\frac{\Gamma^{*}}{2 \pi} \frac{\mathrm{e}^{-|k|} \sinh (k d)}{k\left[c_{v}-c(k)\right]+\mathrm{i} \alpha}\right] \mathrm{e}^{\mathrm{i}(k x-\sigma t)-\alpha t} \mathrm{~d} k\right\} .
$$

The response to the forcing also differs from that of the steady solution found in (3.9). Instead, we obtain

$$
\eta_{p}(x, t)=-\frac{\Gamma^{*}}{\pi} \operatorname{Re}\left\{\int_{0}^{\infty} \mathrm{e}^{\mathrm{i} k\left(x-c_{v} t\right)} \frac{\mathrm{e}^{-k} \sinh (k d)}{k\left[c(k)-c_{v}\right]-\mathrm{i} \alpha} \mathrm{d} k\right\},
$$

where Re stands for taking the real part of the expression. Examination of figure 2 indicates that the equation $c(k)=c_{v}$ has only one solution for $k>0$, which we denote 
$\tilde{k}$. For small $\alpha$ the integrands in (3.39) and (3.40) have one pole

$$
k=\tilde{k}-\frac{\mathrm{i} \alpha}{c_{v}-c_{g}(\tilde{k})}, \quad \alpha \rightarrow 0 .
$$

Note that since $c(k)>c_{g}(k)$ for any $k \neq 0, c_{v}-c_{g}(\tilde{k})>0$. Thus, by adding a small amount of dissipation to the problem we push the pole off the real axis and can obtain a unique solution in the limit that this dissipation goes to zero. The integrals in (3.39) and (3.40) are evaluated using the residue theorem, and we find that the steady response is different upstream, $0<x<c_{v} t$, than downstream, $x>c_{v} t$, of the vortex. For example, for $x-c_{v} t<0$ the particular solution to (3.40) is

$$
\eta_{p}(x, t)=2 \Gamma^{*} \frac{\mathrm{e}^{-\tilde{k}} \sinh (\tilde{k} d)}{c_{v}-c_{g}} \sin \left[\tilde{k}\left(x-c_{v} t\right)\right]-\frac{\Gamma^{*}}{\pi} \operatorname{Re}\left\{\int_{0}^{\infty} \frac{\mathrm{i}^{\mathrm{i} k} \sin (k d) \mathrm{e}^{k\left(x-c_{v} t\right)}}{k c_{v}-\mathrm{e}^{\mathrm{i} k} \sin (k)} \mathrm{d} k\right\},
$$

whereas for $x-c_{v} t>0$

$$
\eta_{p}(x, t)=\frac{\Gamma^{*}}{\pi} \operatorname{Re}\left\{\int_{0}^{\infty} \frac{\mathrm{ie}^{-\mathrm{i} k} \sin (k d) \mathrm{e}^{-k\left(x-c_{v} t\right)}}{k c_{v}-\mathrm{e}^{-\mathrm{i} k} \sin (k)} \mathrm{d} k\right\} .
$$

The first term in (3.42) corresponds to a sinusoidal wave propagating with speed $c_{v}$. The second term decays with distance from the point vortex. Asymptotically expanding the integrals in (3.42) and (3.43) for $\left|x-c_{v} t\right| \gg 1$ we find that their behaviour to leading order is like $O\left(\left|x-c_{v} t\right|^{-2}\right)$. Thus, far upstream of the point vortex one observes a monochromatic wavetrain, while far downstream of the vortex the interface is quiescent. Similarly, we can evaluate the homogeneous solution where a steady response plus a transient solution is obtained for $x<0$ and only a transient solution is obtained for $x>t$. The full linear response, neglecting the transient, is then

$$
\eta(x, t)=2 \Gamma^{*} \frac{\mathrm{e}^{-\tilde{k}} \sinh (\tilde{k} d)}{c_{v}-c_{g}} \sin \left[\tilde{k}\left(x-c_{v} t\right)\right] U\left(t-x / c_{v}\right) U(x),
$$

where $U(t)$ is the Heaviside function. The solution of (3.44) corresponds to the monochromatic wavetrain of wavenumber $\tilde{k}$ which forms upstream but not downstream of the point vortex. The wavetrain extends over an increasing extent of the interface, from near $x=0$ to $x=c_{v} t$. This solution is valid as long as the vertical motion of the point vortex is not $O(1)$ for long times. In $\S 4$, however, we show that for large $t$ the vertical motion of the vortex due to the sinusoidal wavetrain becomes significant and in $\$ 5$ we show numerically that a transition from linear to nonlinear behaviour occurs over long times.

\subsection{The fast point vortex lying close to the wall}

In this section, we consider the effect of a point vortex which is initially placed very close to the wall $\left(Y(0)=O\left(\epsilon^{1 / 2}\right)\right)$ where $0<\epsilon \ll 1$ and which propagates quickly downstream $\left(c_{v}=\tilde{\Gamma} /(4 \pi Y(0)) \gg 1\right)$. This limiting case of strong vortex interaction with the wall differs from the two cases considered above in that we allow the velocity field induced by the point vortex on the interface to be large. We will show that in this case the amplitude of the interface disturbance is independent of the vortex strength. Moreover, because the vortex propagates quickly the motion of the vortex due to the layer is negligible, as is shown in $\S 4$, and the expansion for the vortex position is uniformly valid in time. 
We introduce

$$
\begin{gathered}
Y(0)=\epsilon^{1 / 2} d^{*}, \\
\tilde{\Gamma}=\epsilon^{m} \Gamma^{*}
\end{gathered}
$$

and

$$
c_{v}=\epsilon^{(m-1 / 2)} c_{v}^{*},
$$

where $d^{*}, \Gamma^{*}$, and $c_{v}^{*}$ are $O(1)$, and $-\infty<m<1 / 2$. We assume that the interface is initially either weakly perturbed or unperturbed so that for at least small times the velocity induced on the interface is mainly due to the vortex. We focus our attention on the neighbourhood of the vortex and we introduce the variable $\xi=x-c_{v} t$. This is equivalent to a frame of reference moving with the speed of the vortex, $c_{v}$. As a result, the velocity field in (2.3) can be expressed as

$$
u-\mathrm{i} v=\frac{\epsilon^{(1 / 2+m)} \mathrm{d} \Gamma^{*}}{\pi(\xi+\mathrm{i} y)^{2}} .
$$

This suggests that the velocity field induced on the interface is $O\left(\epsilon^{(1 / 2+m)}\right)$. Taking the real and imaginary parts of (3.48) we find

$$
u^{*}=\frac{\Gamma^{*} d^{*}\left(\xi^{2}-y^{2}\right)}{\pi\left(\xi^{2}+y^{2}\right)^{2}}
$$

and

$$
v^{*}=\frac{2 \Gamma^{*} d^{*} y \xi}{\pi\left(\xi^{2}+y^{2}\right)^{2}},
$$

where $(u, v)=\epsilon^{(1 / 2+m)}\left(u^{*}, v^{*}\right)$. To leading order the kinematic condition in terms of the similarity variable $\xi$ is

$$
v^{*}=-\frac{c_{v}^{*}}{\epsilon} \frac{\partial H}{\partial \xi} .
$$

This suggests that $H(x, t)=1+\epsilon \eta(x, t)$ and implies that disturbances to the interface which are moving with the vortex are $O(\epsilon)$. The solution is obtained by integrating (3.51), yielding

$$
\eta(x, t)=4 d^{*^{2}}\left(\frac{1}{\xi^{2}+1}\right) .
$$

This solution indicates that an $O(\epsilon)$ interface response occurs when $Y \sim O\left(\epsilon^{1 / 2}\right)$.

Note that the amplitude of the disturbance is independent of the strength of the vortex and depends solely upon the proximity of the point vortex to the wall. This can be interpreted by a simple dimensional argument. When the point vortex is close to the wall, the vortex and its image induce a rate of deformation on the interface which is proportional to $(2 \Gamma d) / H_{\infty}^{2}$. However, the duration of the forcing on a particular sector of the interface is proportional to $\left(d H_{\infty}\right) / \Gamma$. The deformation of the interface is the product of the induced velocity, $(2 \Gamma d) / H_{\infty}^{2}$, and the duration, $\left(d H_{\infty}\right) / \Gamma$, that the velocity acts on the interface. Hence, the amplitude of disturbances on the vortex layer depends solely upon how close the point vortex is to the wall.

In addition to (3.52) there is also a transient disturbance. Analysis of the velocity field for $|\xi| \gg 1$ shows that the velocity induced by the vortex is $O\left(\epsilon^{(3 / 2-m)}\right)$ and so 
the contribution of the vortex layer (the homogeneous solution, as discussed in §3) becomes dominant. As a result, after the vortex has passed, dispersive transient waves remain. We note that there must be some waves that remain because the area of the disturbance described by (3.52) is positive and consequently the area displaced by the dispersive waves far upstream of the vortex must cancel that propagating in tandem with the vortex.

We find that two disturbances are created on the interface a non-dispersive wave, which propagates rapidly in tandem with the point vortex, and a disturbance which is left behind the vortex and slowly disperses. Moreover, because the vortex is moving very quickly from the dispersive waves it can be shown that their effect on the motion of the vortex will be negligible.

\section{Conservation of momentum}

In the cases studied in the previous two sections, we assumed that disturbances on the interface do not influence, to leading order, the motion of the point vortex. We then verified that this assumption was valid for the non-resonant cases $\left(c_{v} \ll 1\right.$ and $c_{v} \gg 1$ ). In this section, we derive a general relationship between the vertical displacement of the vortex and the norm of disturbance amplitude on the interface. We verify the results of the previous sections for the motion of the point vortex and show that, in the case where the vortex resonates with the interface, the long-time vertical motion of the vortex is not negligible. Finally, for a small disturbance we obtain a bound on the vertical motion of the vortex.

Following Bell (1990), we apply conservation of the $x$-component of momentum to a region, $R^{2}$, containing the point vortex and the vortex layer. The momentum (see Batchelor 1967, pp. 529-530) is defined as

$$
P=\int_{R^{2}} y \omega \mathrm{d} A,
$$

where $\omega$ is the vorticity, which here (non-dimensionalized) is equal to 1 within the vortex layer and where we integrate over the perturbed part of the interface (since the unperturbed interface only adds a constant value to the momentum). Thus, we write

$$
P=\tilde{\Gamma} Y(t)+\int_{-\infty}^{\infty} \int_{1}^{H(x, t)} y \mathrm{~d} y \mathrm{~d} x,
$$

where $H(x, t)=1+\eta(x, t)$. Using the invariance of momentum and applying (4.2) we find

$$
Y(t)-Y(0)=-\frac{1}{2 \tilde{\Gamma}}\left(\|\eta\|^{2}-\left\|\eta_{o}\right\|^{2}\right),
$$

where we have assumed that $\|\eta(x, t)\|^{2}=\int_{-\infty}^{\infty}(\eta(x, t))^{2} \mathrm{~d} x$ is bounded. This relation shows that the point vortex transfers momentum to the interface which both transfers this momentum downstream by forming dispersive waves and transfers it back to the point vortex resulting in vertical motion. When the interaction between the vortex and the layer is weak the momentum is carried to the far-field via dispersive waves. Thus, when eruption occurs we would expect that very little momentum gets carried away by waves and most of the momentum is transferred back to the vortex resulting in a strong interaction. Not surprisingly, this relation is unchanged if we neglect the effect of the wall. We also note that for $\tilde{\Gamma}=0$ we find $\|\eta\|^{2}=\left\|\eta_{o}\right\|^{2}$ which gives 
a bound on the growth of an initial disturbance (Dritschel 1988b). Equation (4.3) allows us to determine the vertical trajectory of the point vortex from the norm of the interface. Although calculating an exact analytical expression for the interface is impossible in most cases, we can deduce the direction of the vertical motion of the point vortex from this expression. For example, if $\tilde{\Gamma}>0$ then the motion of the point vortex will be downward. Whereas if $\tilde{\Gamma}<0$ then the path of the point vortex will be upward.

\subsection{The linear limit}

We consider interface disturbances which are small such that we can decompose the interface solution into a particular and a homogeneous solution. Assuming a small disturbance to an initially flat interface, (4.2) becomes

$$
Y(t)-Y(0)=-\frac{1}{2 \tilde{\Gamma}}\left(\left\|\eta_{h}+\eta_{p}\right\|^{2}\right),
$$

where $\eta_{h}$ is the homogeneous solution and $\eta_{p}$ is the particular solution. For the travelling wave solutions found in $\S \S 3$ and $4, \eta_{p}=f\left(x-c_{v} t\right)$ and we have

$$
\frac{\mathrm{d}}{\mathrm{d} t}\left\|\eta_{p}\right\|^{2}=0
$$

Observing that $\eta_{p}(x, 0)=-\eta_{h}(x, 0)$ and applying (4.5) we find $\left\|\eta_{p}(\xi)\right\|^{2}=\left\|\eta_{h}(\xi)\right\|^{2}$ where $\xi=x-c_{v}$. Applying this result and the Schwarz inequality to (4.4), an upper bound on the vertical motion of the point vortex due to any small-amplitude disturbance can be obtained yielding

$$
|Y(t)-Y(0)| \leqslant \frac{2}{|\tilde{\Gamma}|}\left(\left\|\eta_{p}\right\|^{2}\right) .
$$

A stronger statement can be made for the cases $c_{v} \ll 1$ and $c_{v} \gg 1$ in the limit as $t \rightarrow \infty$. In these cases, the solution due to the point vortex, the particular solution, and the solution due to the vortex layer, the homogeneous solution, will separate from one another as $t \rightarrow \infty$ (see e.g. figure 10) implying that the cross-product term on the right-hand side of (4.4) is zero. As a result, in the limit as $t \rightarrow \infty$

$$
|Y(t)-Y(0)| \leqslant \frac{1}{|\tilde{\Gamma}|}\left(\left\|\eta_{p}\right\|^{2}\right) .
$$

For example, using the particular solution (3.36), in \$3.3.3, together with the above result, reproduces the solution (3.34) for the terminal vertical position of the point vortex.

When the vortex resonates with the interface, $0<c_{v}<1$, (3.44) shows that an expanding interval of monochromatic travelling waves is left upstream of the vortex. Since the norm of $\eta(x, t)$ grows as the horizontal extent of the waves grow in time, the displacement of the point vortex from its initial position will grow with time. For example, if $\tilde{\Gamma}<0(\tilde{\Gamma}>0)$ then the vortex will be attracted to (repelled from) the interface. As a result, for $\tilde{\Gamma}<0$ the disturbances to the interface will grow in time. However, because the vertical speed of the point vortex is slow compared to the speed of the interfacial waves the travelling wave solution given in (3.44) can be seen for $O(1)$ times (see figures 5 and 7). For large $t,(4.3)$ predicts the vortex will move an $O(1)$ distance and change the character of the solution from linear to nonlinear. 


\section{Numerical solutions}

The analytical solutions presented in $\$ 3$ correspond to asymptotic cases wherein the interface departs slightly from a mean position and the motion of the vortex is uncoupled, at leading order, from that of the interface. However, the motion of the interface is coupled to that of the vortex. Such solutions cannot represent the general evolution of the interface where there is a strong mutual interaction between the interface and the vortex. In this section, we first compare the linear theories developed in $\$ 3$ with the numerical results of the full nonlinear problem. We then examine certain nonlinear behaviour and interpret a wide array of the observed phenomena within the context of the linear theories. In addition, we illustrate that linear behaviour can transition to nonlinear behaviour due to the motion of the point vortex leading to the ejection of vorticity away from the wall.

To track the time evolution of the position $(x(t), y(t))$ of Lagrangian particles, (2.3) and (2.5) must be integrated spatially followed by the temporal integration of

$$
\mathrm{d} x / \mathrm{d} t=u
$$

and

$$
\mathrm{d} y / \mathrm{d} t=v .
$$

The numerical method employed is similar to Pullin (1981, see also Stern \& Pratt 1985), except that we consider a finite non-periodic domain subject to the forcing of a localized point vortex. The spatial integration of (2.3) and (2.5) was carried out using the trapezoidal rule and the time integration was done using a second-order Adams-Bashforth predictor-corrector scheme. To significantly save computation time an analytic solution for the influence of the unperturbed vortex layer is used, leaving only the perturbed interface to be calculated numerically. The spatial domain was adjusted such that when an endpoint exceeded the height of the unperturbed interface by more than $10^{-6}$, a point was added to lengthen the computational domain. To obtain an error estimate we computed the circulation and the $x$-component of momentum, both of which should be invariant over time.

\subsection{Weak point vortex outside the layer}

For a weak point vortex $(\tilde{\Gamma}=0.05)$, located outside the layer $(X(0)=0, Y(0)=2.5)$, and an initially flat unperturbed interface, figure 3 shows the interface response at six times. As expected, the vortex initially exerts a downward force on those points upstream of the vortex $(x<0)$ and an upward force on those points downstream of the vortex $(x>0)$. Thus, a profile which is nearly antisymmetric about the $x$ location of the vortex is initially formed with a negative hump slightly upstream of the origin $(x<0)$ and a positive hump slightly downstream of the origin $(x>0)$. As time progresses, the magnitudes of these humps increase and reach the level of the steady solution predicted by the theory. The negative hump corresponding to the travelling wave maintains its form, while the positive hump disperses with long waves propagating to the right faster than short waves (see (3.10)). We also note that although the transient solution decays quickly, $O\left(1 / t^{(d-1) / 2}\right)$, its effects persist over large distance, $x=O(t)$.

Figure 4(a) shows the vertical displacement of a point vortex for the case described above. The vortex moves slightly downward and then oscillates about a new steadystate height which corresponds to the value predicted by (3.34). The oscillations have a period which can be seen to be nearly $4 \pi$, corresponding to a frequency of $1 / 2$. The amplitude of the oscillations decays corroborating the linear theory, 


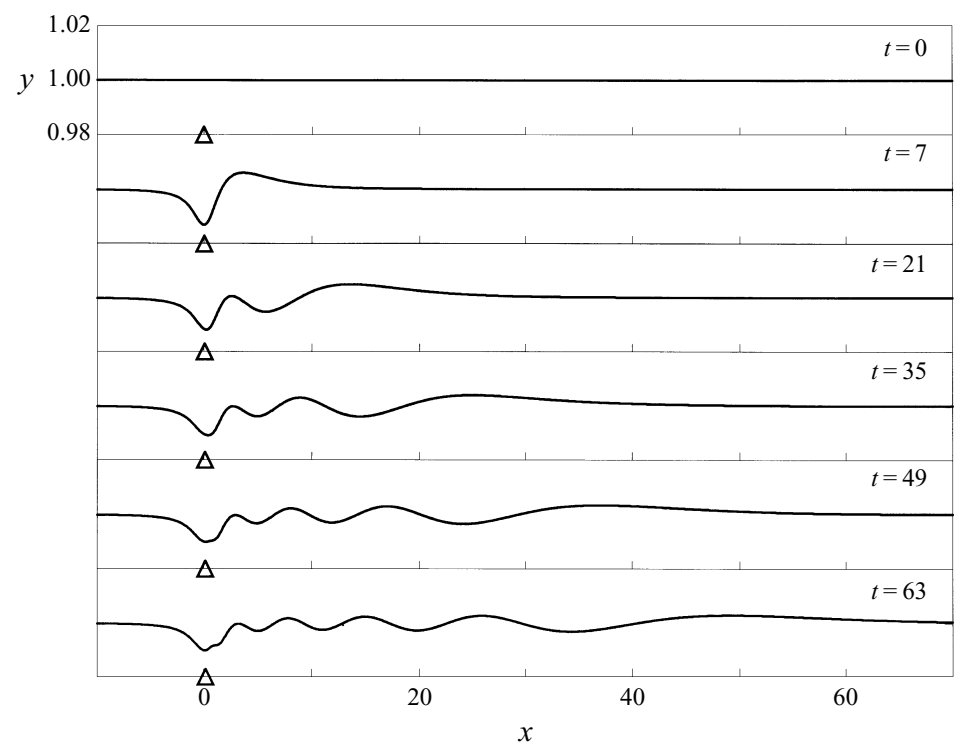

Figure 3. The evolution of an initially flat interface subject to the forcing of a point vortex, $\tilde{\Gamma}=0.05$, which is initially located at $X(0)=0$ and $Y(0)=2.5$ for six times $0 \leqslant t \leqslant 63$. Though the vortex lies above the interface and is not shown in the figure, see figure 4 , its $x$-location is indicated by an open triangle. As time evolves the interface forms a steady solution near $x=0$ superposed with dispersive waves where the long waves travel faster downstream than the short waves. Note that the amplitude of these waves decays as they propagate downstream.
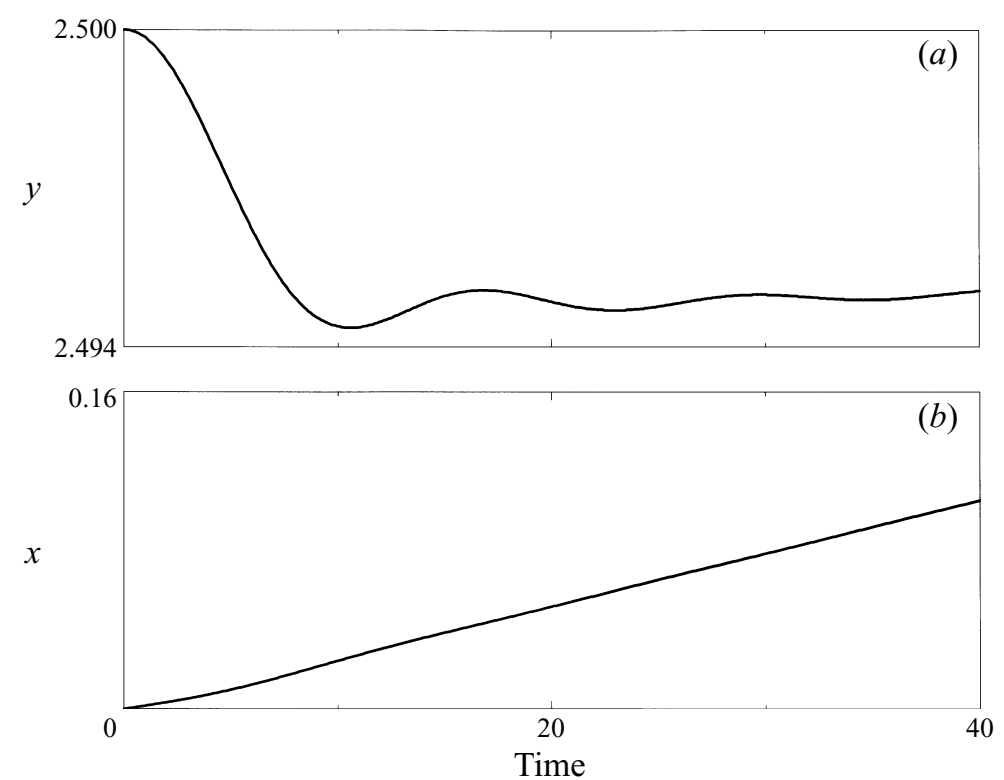

FiguRE 4. The position $(X(t), Y(t))$ of the point vortex for the case shown in figure 3. $(a)$ The vertical displacement of the point vortex versus time. The vortex initially moves monotonically downward and then undergoes decaying oscillations of period $4 \pi$ about a steady-state height. $(b)$ The horizontal displacement of the point vortex versus time. For time $t<10$ the horizontal velocity has a slight time dependence, but then relaxes to a uniform horizontal velocity given by $\tilde{\Gamma} /(4 \pi(d-1))$. 
which assumes that the leading-order vertical position of the point vortex is timeindependent. Comparing the time-dependent motion of the interface near $x=0$ (see (3.21)) with the time-dependent vertical motion of the vortex in figure $4(a)$, we see that both undergo oscillations with a frequency close to $1 / 2$. This implies that a coupling exists between the short waves and the vortex. This coupling becomes stronger when the vortex is close to the layer and, eventually, if the vortex is initially placed close enough to the interface a breakdown in the linear theory will occur.

Figure $4(b)$ shows the horizontal motion of the point vortex. This motion of the vortex is transient for short times but then quickly relaxes to the uniform velocity $c_{v}=\tilde{\Gamma} /(4 \pi(d-1))$.

\subsection{Weak point vortex inside the layer}

In $\$ 3.4$ we showed that by linearizing (2.3)-(2.6), with the vortex speed $c_{v}$ fixed, a sinusoidal monochromatic wavetrain formed upstream of the vortex when the horizontal velocity of the vortex was equal to the phase velocity of one of the interfacial waves. In $\S 4$, however, we showed that the vertical motion of the vortex could not be ignored and thus the vortex-interface resonant interaction would result in a slow breakdown of the monochromatic wavetrain solution. In this subsection, we present numerical calculations (figures 5-9) of the interface shape and vortex trajectory when the point vortex lies within the layer of constant vorticity and moves with a horizontal speed that coincides with the phase velocity of one of the interfacial waves. We consider three cases and discuss the effect of the motion of the vortex toward or away from the interface as compared to the linear solution obtained in $\$ 3.4$.

We first consider a weak positive point vortex $(\tilde{\Gamma}=0.025)$ that is placed near the middle of the vortex layer $(X(0), Y(0))=(0,0.6)$. While the linear theory predicts that a monochromatic wavetrain of constant amplitude will form upstream of the point vortex where the wavelength of the wavetrain is determined by the condition, $c(\tilde{k})=c_{v}$, figure 5 shows that the interface evolves into a wavetrain that is slowly modulated in both amplitude and wavenumber. Figure 6 shows the movement of the point vortex as a function of time and as predicted in $\S 5(\tilde{\Gamma}>0)$ the vortex moves closer to the wall. As a result, the vortex speed, for large times, increases to leading order. The linear theory suggests that one effect of the change in the horizontal vortex speed is to modify the wavenumber which satisfies the resonance criteria. Thus, as seen in figure 5, the effect of the vertical motion may act not only to modulate the amplitude of the wavetrain but the wavelength as well. Although the large displacement of the vortex violates the linear theory, the movement occurs slowly implying that the linear theory can be applied for $O(1)$ variations from a given time. Thus we would expect a wavetrain of nearly constant amplitude to form whose wavelength slowly grows with time. In this case, due to its vertical displacement, the acceleration of the vortex in the horizontal direction acts to accelerate the growth in extent of the disturbances over the interface. Since the modulated wavetrain is of large extent and does not appear to decay with time, the nature of the interaction may be called 'global'.

We next consider a weak vortex of negative strength $(\tilde{\Gamma}=-0.025)$ placed near the middle of the vortex layer $(X(0), Y(0))=(0,0.4)$. In figure 7 we see that for short times, as for $\tilde{\Gamma}=0.025$ shown in figure 5 , a modulated wavetrain begins to form. However, as is shown in figure 8, the movement of the point vortex is toward the interface and as a consequence its horizontal velocity decreases. This has the effect of slowing the expansion of the wavetrain and, as seen in figure 7 for $t=60.0$, causing the envelope of the wavetrain to focus and grow in amplitude. 


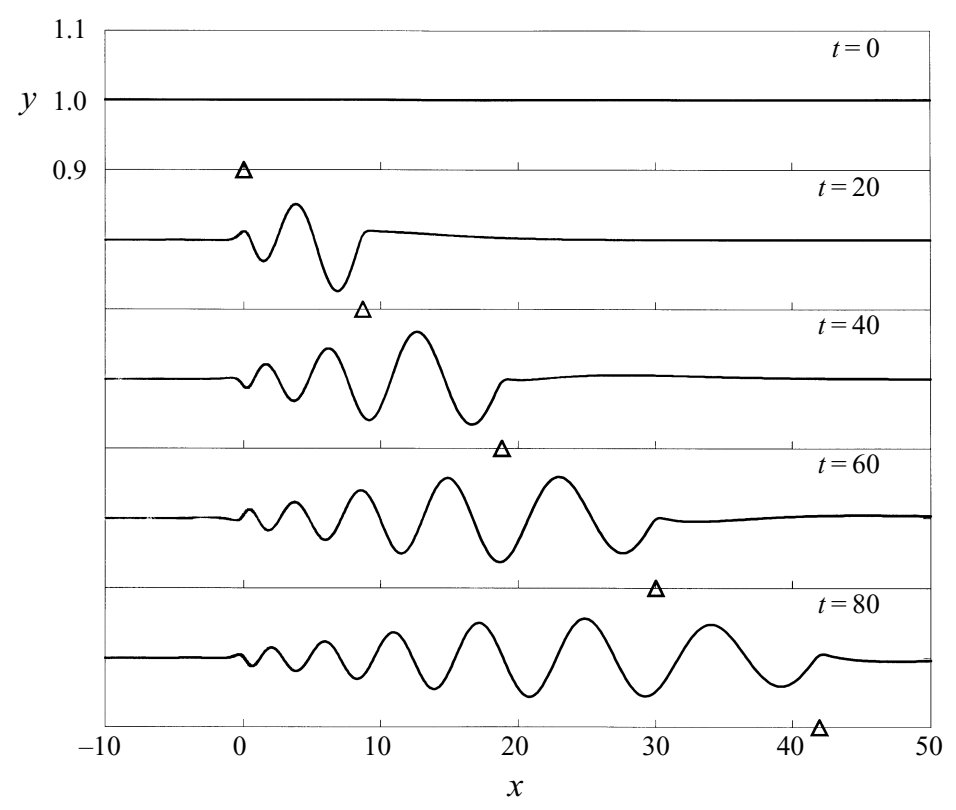

FIGURE 5. The evolution of an initially flat interface subject to the forcing of a weak point vortex, $\tilde{\Gamma}=0.025$, which is initially located at $X(0)=0$ and $Y(0)=0.6$ for five times $0 \leqslant t \leqslant 80$. Though the vortex lies above the interface and is not shown in the figure, see figure 6 , its $x$-location is indicated by an open triangle. A modulated envelope forms behind the point vortex due to its resonant interaction with the interface. The extent of the interface which is covered by the expanding envelope is proportional to time and the speed of the vortex.
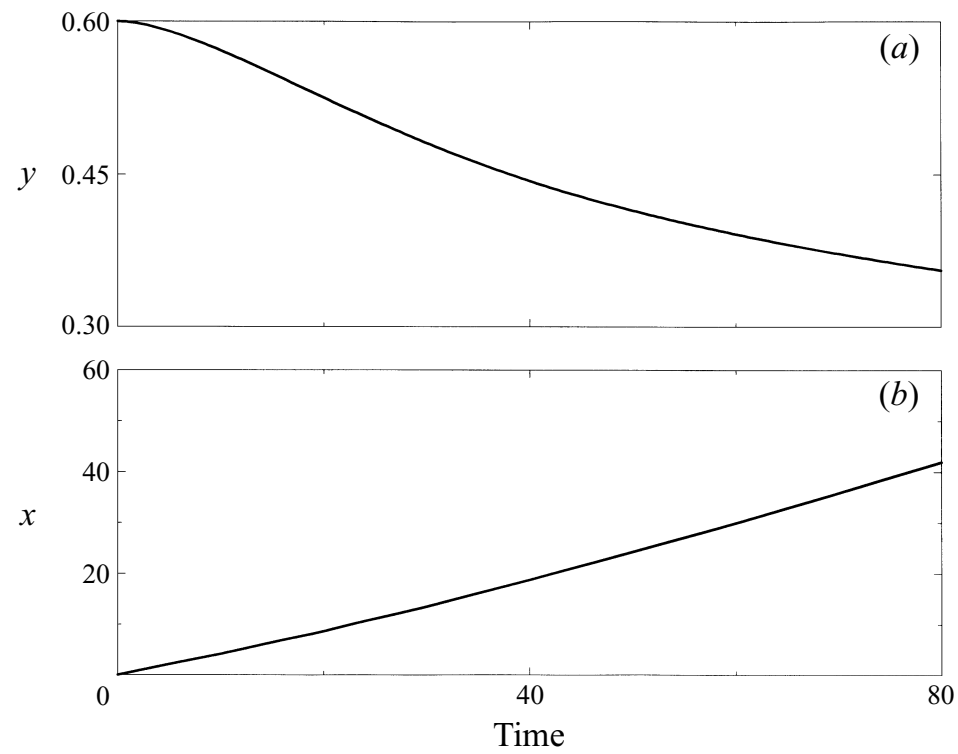

Figure 6. The position $(X(t), Y(t))$ of the point vortex for the case shown in figure 5. $(a)$ The vertical displacement of the point vortex versus time. The vortex moves monotonically toward the wall in time but its vertical speed decreases as it moves further from the interface. $(b)$ The horizontal displacement of the point vortex versus time. As the vortex moves closer to the wall, its horizontal speed increases monotonically. 


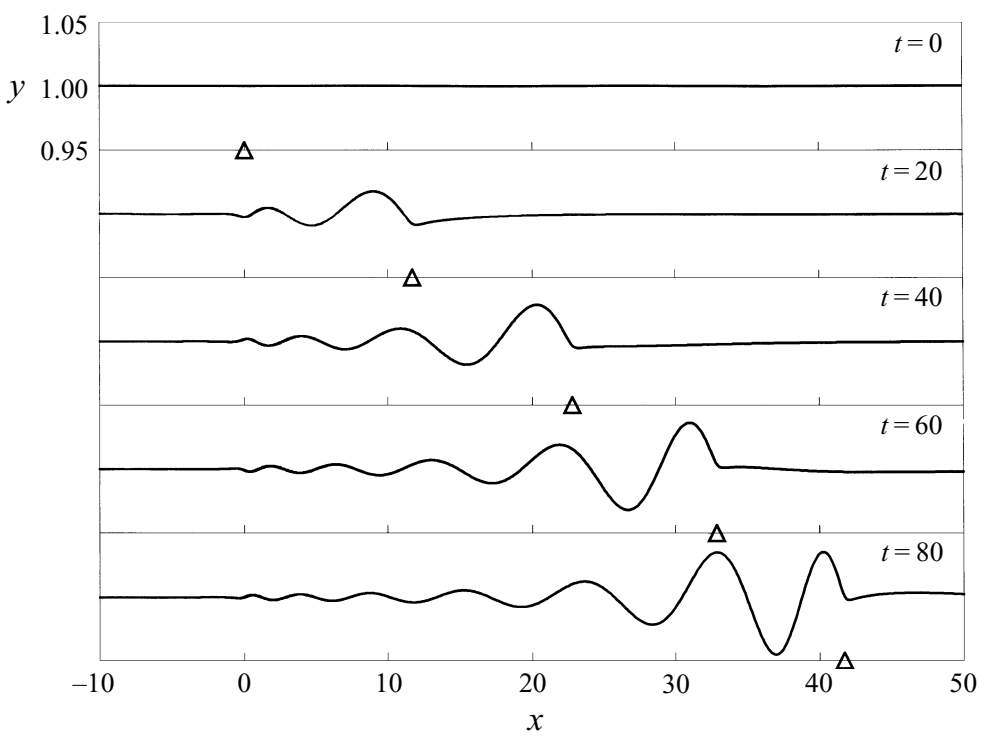

FIGURE 7. The evolution of an initially flat interface subject to the forcing of a weak point vortex of negative strength, $\tilde{\Gamma}=-0.025$, which is initially located at $X(0)=0$ and $Y(0)=0.4$ for five times $0 \leqslant t \leqslant 80$. Though the vortex lies above the interface and is not shown in the figure, see figure 8 , its $x$-location is indicated by an open triangle. A modulated wave packet begins to form due to the resonant interaction between the point vortex and the interface. However, as time progresses the envelope of the wavetrain focuses into a narrow region near the front of the wavetrain. The extent of the interface which is covered by the expanding envelope is proportional to time and the speed of the vortex.
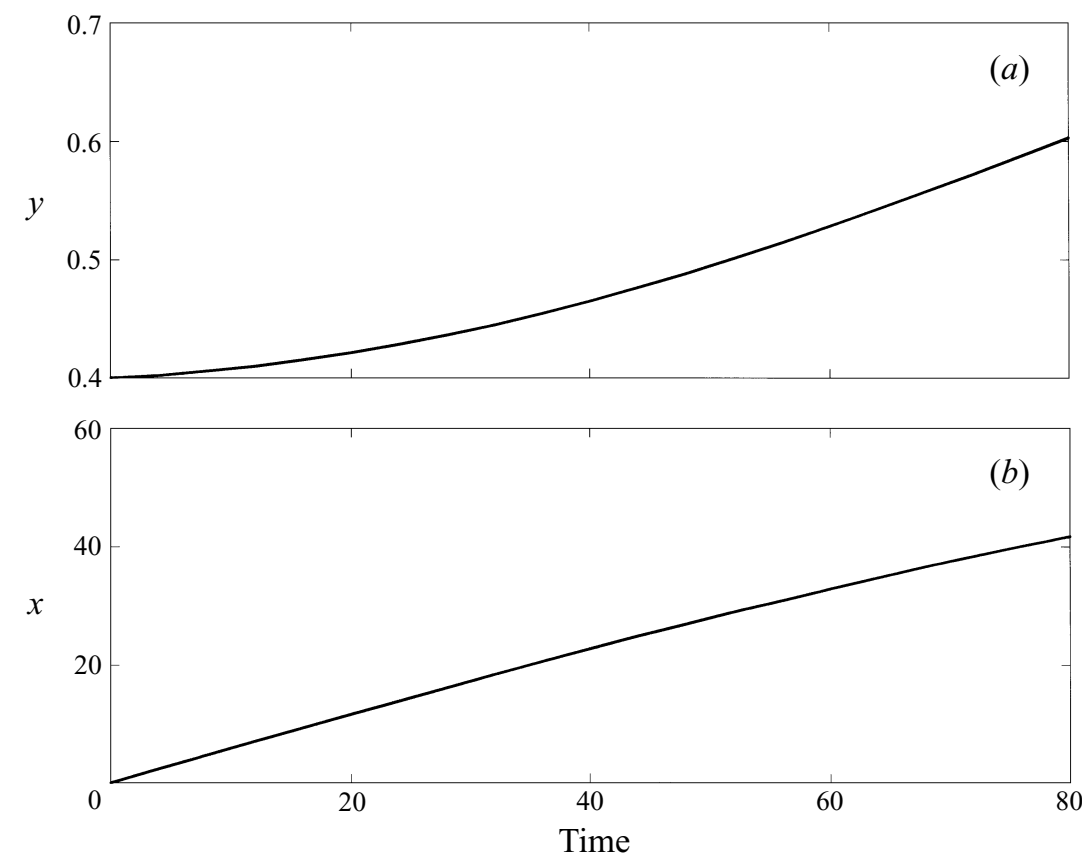

Figure 8 . The position $(X(t), Y(t))$ of the point vortex for the case shown in figure 7. $(a)$ The vertical displacement of the point vortex versus time. The vortex moves monotonically toward the interface in time. (b) The horizontal displacement of the point vortex versus time. Its horizontal speed decreases monotonically as it moves upward toward the interface. 


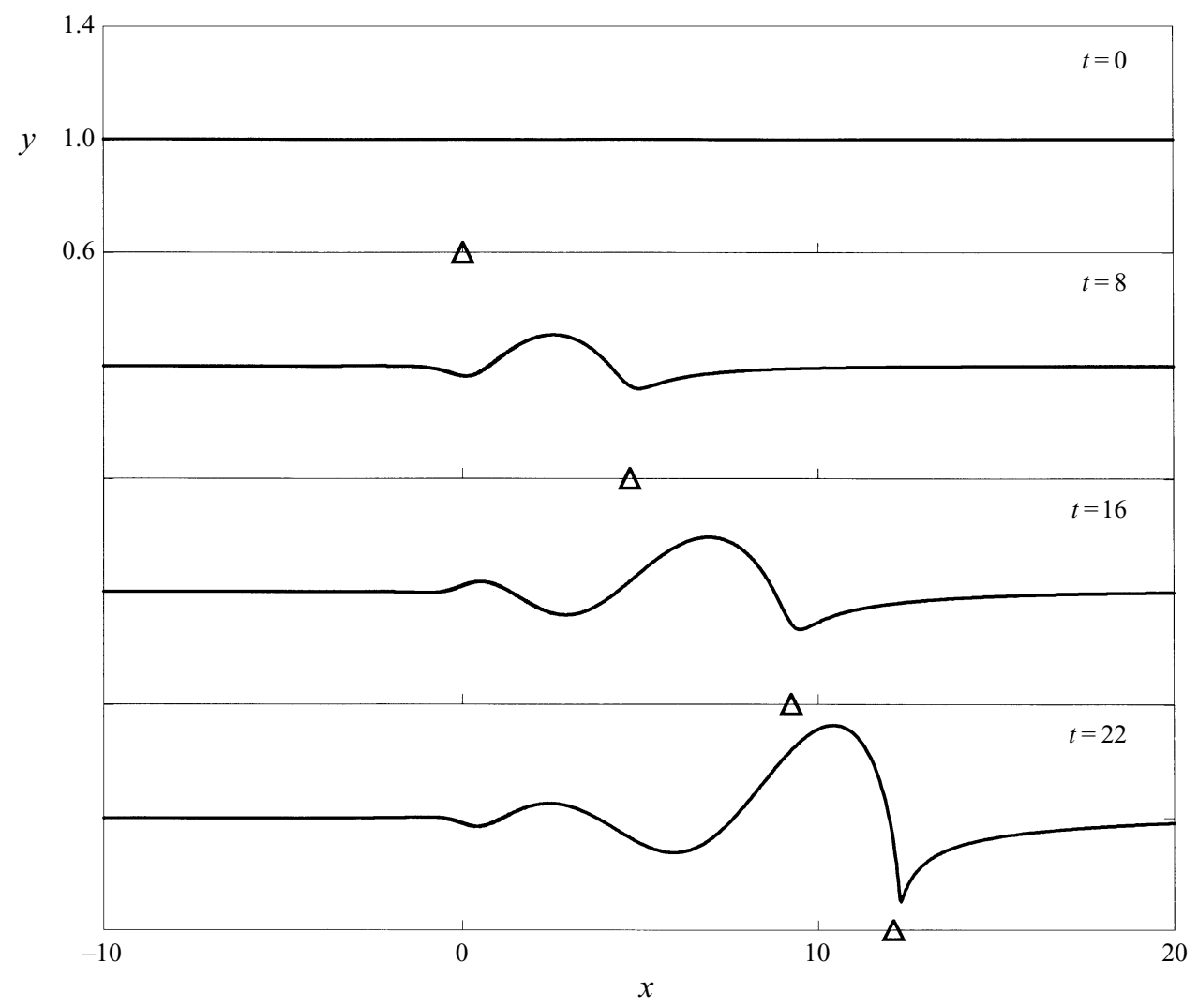

FIGURE 9. The evolution of an initially flat interface subject to the forcing of a point vortex of strength $\tilde{\Gamma}=-0.5$, which is initially located at $X(0)=0$ and $Y(0)=0.2$ for four times $0 \leqslant t \leqslant 22$. The vortex is off the scale of the figure, $(X(22), Y(22))=(12.1,0.54)$. The open triangle denotes the $x$-location of the point vortex. A modulated wavetrain starts to form due to the resonant interaction between the point vortex and the interface but because of the upward displacement of the point vortex and its strength, large-amplitude disturbances form. The disturbance appears as a region of vorticity which is ejected into the irrotational flow.

In figure 9, we consider a stronger vortex of negative strength $(\tilde{\Gamma}=-0.5)$ initially placed closer to the wall $(Y(0)=0.2)$. In this case, we again see that the vortex drifts toward the interface, slowing down in the process. However, in this case the amplitude of the disturbances are large and grow too quickly, due to the motion of the vortex toward the interface and its $O(1)$ strength, for the slowly modulated wavetrain to develop. Instead, we see that a large-amplitude narrowing region of vorticity, located almost directly above the vortex, is ejected into the irrotational flow.

\subsection{The fast vortex near the wall}

In figure 10 a strong vortex is initially placed close to the wall, $\tilde{\Gamma}=10.0,(X(0), Y(0))=$ $(0,0.1)$, so that its velocity is fast. Note the greater extent needed for the $x$-scale compared with the previous cases. The figure shows a non-dispersive wave propagating downstream in conjunction with the vortex and leaving behind it dispersive transient waves. Initially the dispersive waves are the same amplitude as the non-dispersive wave but as $t$ becomes large they decay and their amplitude becomes much smaller. Furthermore, in agreement with the asymptotic theory, the amplitude of the waves 


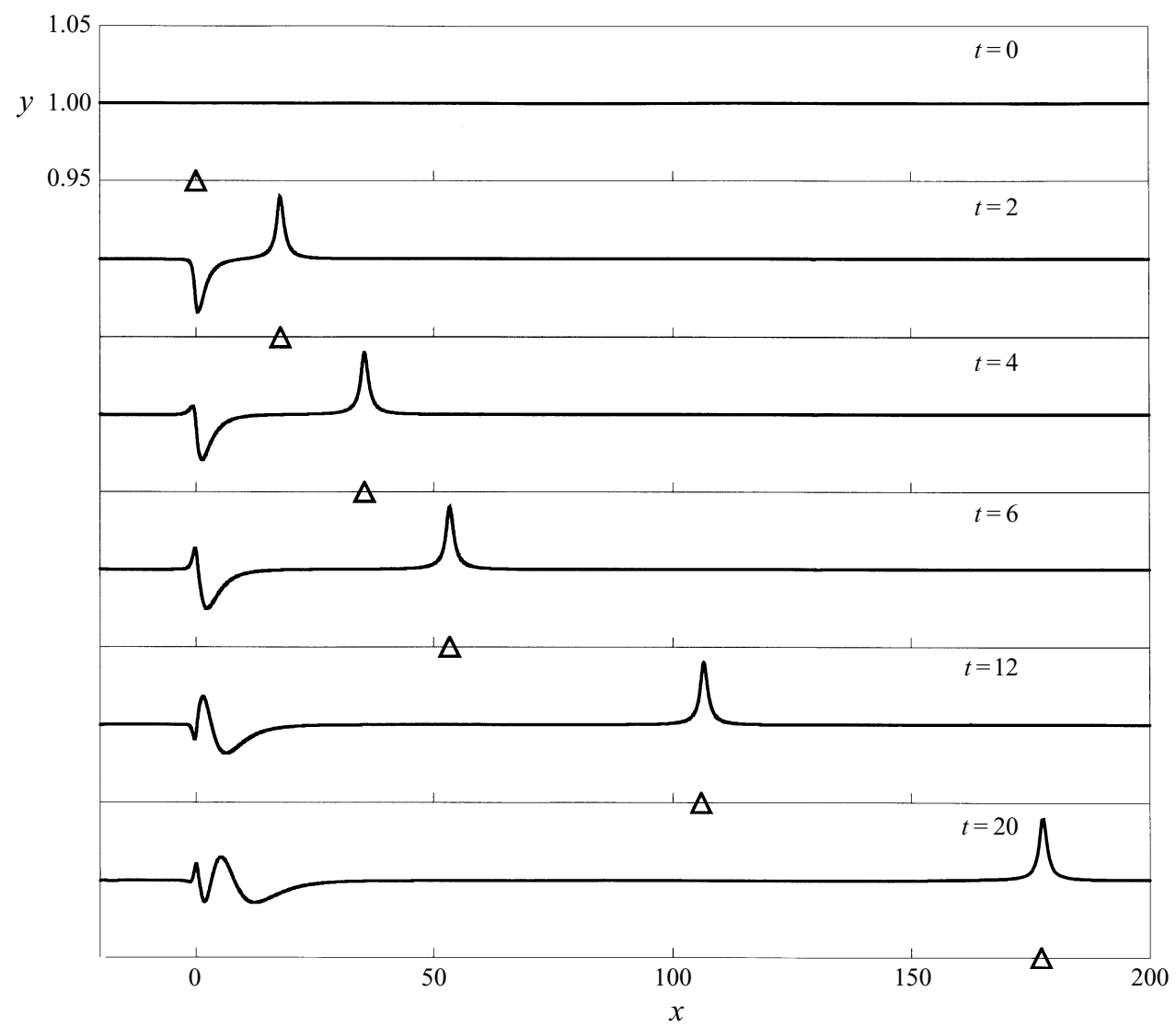

FIGURE 10. The evolution of an initially flat interface subject to the forcing of a strong point vortex, $\tilde{\Gamma}=10.0$, which is initially located at $X(0)=0$ and $Y(0)=0.1$ for six times $0 \leqslant t \leqslant 24$. Note the greater extent needed for the $x$-scale compared with the previous cases. A non-dispersive wave propagates downstream in conjunction with the vortex and leaves behind it dispersive, transient waves. The vortex is off the scale of the figure and is located directly below the non-dispersive wave. The open triangle denotes the $x$-location of the point vortex.

are small despite the strong forcing. The theory predicts that the amplitude of the non-dispersive disturbance is proportional to $4 d^{2}$ and this is corroborated by the numerical solutions shown in figure 10. As in the slow vortex case the solution is the superposition of the forcing due to the point vortex and dispersive transient waves. However, in this case, the time-independent solution separates itself from the dispersive waves because $c_{v}$ is much larger than any $c(k)$.

\section{Discussion and conclusions}

We have examined the mutual interaction of a point vortex with a wall-bounded layer of constant vorticity as a model for unsteady vortex-boundary layer interactions. The point vortex may lie outside the layer of constant vorticity or it may lie within the layer which then acts to convect it downstream. In the examples presented here, the vortex layer was initially of uniform thickness. The point vortex acts to deform the vortex layer while the disturbed layer induces a velocity both on itself, furthering its deformation, as well as on the point vortex, altering its trajectory. When the motion of 
the point vortex does not depend, to leading order, on the evolution of the interface and disturbances are small, a linear theory can be derived to describe the motion of the interface and the point vortex. In this case, a variety of behaviours are shown to exist which include dispersive waves, monochromatic wavetrains and compact waves of unchanging form. The horizontal extent of the monochromatic wavetrain increases at a rate $c_{v}$ resulting in a disturbance of large horizontal extent. Thus despite the highly localized character of the point vortex it can initiate non-local disturbances. As the monochromatic wavetrain propagates downstream, the vertical displacement of the point vortex may become large. This motion violates an assumption of the linear theory and acts to slowly modulate both the amplitude and wavenumber of the wavetrain.

For a weak vortex lying outside the layer, a steady solution superposed with a dispersive disturbance whose amplitude decays in time is observed. In this case, the long-time solution approaches a steady state. As a result, we can match the short-time solution, which neglects the motion of the point vortex, to the long-time solution, which accounts for the motion of the vortex, and obtain a solution for the interface motion that is uniformly valid (3.37). Furthermore, we see from (3.21) that the long-time decay of the disturbances near the vortex $\left(x \approx x_{v}\right)$ depends upon the initial proximity of the vortex to the interface. Also, the amplitude of interfacial disturbances depends upon the vortex strength relative to the vortex layer, $\tilde{\Gamma}$. Thus, a transition to finite-amplitude disturbances can occur only by either increasing the strength of the vortex or by moving the initial height of the vortex closer to the interface. However, it cannot occur because the vortex moves a large distance from its initial position toward the layer.

A weak vortex propagating inside the layer with speed $c_{v}$ is resonant with the interfacial waves of speed $c(\tilde{k})=c_{v}$. As a result, a modulated wavetrain is produced on the interface whose extent ranges from near $x=0$ to $x=c_{v} t$. The perturbed interface induces a vertical displacement on the point vortex, causing it to be repelled from or attracted to the interface. The vertical motion of the point vortex is slow and so for $O(1)$ times the linear theory remains valid. For long times, however, the point vortex has moved an $O(1)$ distance and the assumption that the height of the point vortex is constant is no longer valid. Thus, accounting for the vertical displacement of the point vortex is important for ascertaining whether the system eventually reaches a steady state (as in the case of a weak vortex outside the layer) or whether a transition to another, perhaps nonlinear, time-dependent state occurs. Whether the point vortex is attracted to or repelled by the interface depends upon the sign of $\tilde{\Gamma}$. Recall that in the frame of reference used here, the undisturbed velocity in the vortex layer increases linearly from 0 at the interface to 1 at the wall. For $\tilde{\Gamma}>0$, the vortex is attracted toward the wall and is thus convected at a higher speed which accelerates the rate at which the wavetrain spreads over the interface. For $\tilde{\Gamma}<0$, the vortex is repelled from the wall into a level of lower velocity, slowing the vortex and consequently focusing the interaction into a narrow region. This region grows in amplitude, and so appears as vortical fluid being ejected into the irrotational fluid above.

We have interpreted the effect of the motion of the weak vortex as enacting a slow modulation in space and time on the monochromatic wavetrain solution. The nonlinear Schrödinger (NLS) equation has been derived for the slow modulation of wavepackets in a wall-bounded layer of constant vorticity (see Balagondar, Maslowe \& Melkonian 1987). Moreover, the NLS equation in some cases is modulationally unstable and this instability acts to modulate a monochromatic wavetrain until the modulated envelope grows into a soliton where nonlinearity and dispersion are 
balanced. As a result, it is tempting to suggest the NLS equation, or a modified version of it containing a forcing term, as a model for the behaviour seen in figures 5 , 7 and 9. However, in the present case, the vertical position of the vortex is coupled to the interface motion and so the velocity field is nonlinear to leading order. As a result, the relationship (or lack thereof) between the behaviour we have seen numerically and that of the well-known NLS equation is unclear.

When a strong vortex is placed close to a wall a steady solution moving in tandem with the point vortex $\left(c_{v} \gg 1\right)$ propagates downstream leaving dispersive waves whose phase speed is $0<c<1$ far behind. In this case, the terminal state is a compact wave which appears steady in a reference frame moving with the vortex. Increasing the strength of the point vortex will increase the rate of growth of disturbances on the vortex layer; concurrently, a stronger vortex will move faster, reducing the period during which rapid growth occurs. These antagonistic effects precisely cancel; consequently, the amplitude of the disturbances are independent of the vortex strength. The case of a fast vortex was considered in a different geometry in Bernoff, van Dongen \& Lichter (1996). There, the vortex was convected by a shear flow above a viscous boundary layer. Steady solutions were found when the vortex is convected at a sufficiently high speed. The results from both Bernoff et al. (1996) and the fast vortex case considered here share the common feature of possessing a steady solution which propagates in tandem with the vortex.

The results presented in this work may usefully describe boundary layer phenomena for which the viscous timescale $T_{v} \sim d^{2} / v$ is small compared with the timescale $T_{\eta} \sim$ $d^{2} / \Gamma$ for the vortex-induced deformation. Consequently, we require that $T_{\eta} / T_{v} \sim$ $v / \Gamma \ll 1$. This criterion is equivalent to requiring that the Reynolds number be large.

Our model relies, as well, on the vortex layer being of constant vorticity. Certainly, more complicated distributions of vorticity will alter the details of the interaction of the point vortex with the layer, as well as the self-interaction of the layer. However, we present this simplified model as a means to understand the mechanisms at work in more complicated cases. For example, as discussed above and in §3.4, the criterion for sustained unsteady interaction is that the speed of the vortex match the phase velocity of an interfacial wave. While a more complicated distribution of vorticity will alter the dispersion relation and hence the phase speeds, the resonance criterion is expected to remain.

A variety of phenomena, such as dispersive waves and the ejection of vorticity, are seen in our model that have been observed in boundary layer flows, indicating that vortex interactions in the presence of a wall may be an important two-dimensional mechanism for the ejection of vorticity and the breakup of the boundary layer into small-scale regions of vortical and irrotational flow. We have seen preliminary numerical evidence for rapid ejection, rollup, entrainment, and the existence of solitary waves. Moreover, we can study the evolution of these phenomena when the thickness of the layer becomes large due to a strong interaction between the point vortex and the interface. A more detailed study of these cases and the parameter regimes for which they exist is the focus of future work.

The financial support of the National Science Foundation, grant CTS-9206828, is gratefully acknowledged. The authors also acknowledge the referee's thorough reading of the manuscript and useful suggestions. S.L. also thanks the NATO Scientific Affairs Division for a grant (CRG.910443) to visit the laboratory of GertJan van Heijst at University of Eindhoven, the Netherlands, where experiments with JanBert Flór on coherent structures interacting with boundaries stimulated this research. 


\section{Appendix}

The second term on the right-hand side of (3.19) can be expressed in terms of

$$
I=\mathrm{e}^{-\mathrm{i} t / 2} \int_{0}^{\infty} \mathrm{e}^{-k(d-1)} \mathrm{e}^{\left.\mathrm{i}\left[k x+\mathrm{e}^{-2 k} t / 2\right)\right]} \mathrm{d} k,
$$

where we have substituted the expression for $c(k)$ given by (3.11). If we let $u=\mathrm{e}^{-2 k}$, then

$$
I=\frac{1}{2} \mathrm{e}^{-\mathrm{i} t / 2} J(\alpha, t)
$$

where

$$
J(\alpha, t)=\int_{0}^{1} u^{\alpha} \mathrm{e}^{\mathrm{i} u t / 2} \mathrm{~d} u
$$

and where $\alpha=(d-3-\mathrm{i} x) / 2$. Note that provided that $\operatorname{Re}(\alpha)>0$, integration by parts gives the functional relation

$$
J(\alpha, t)=\frac{\mathrm{e}^{\mathrm{i} t / 2}}{\mathrm{i}(t / 2)}-\frac{\alpha}{\mathrm{i}(t / 2)} J(\alpha-1, t) .
$$

Therefore it suffices to evaluate $J(\alpha, t)$ for $-1<\operatorname{Re}(\alpha)<0$, yielding

$$
J(\alpha, t)=\frac{2^{(\alpha+1)}}{t^{(\alpha+1)}} \int_{0}^{t / 2} v^{\alpha} \mathrm{e}^{\mathrm{i} v} \mathrm{~d} v .
$$

For large $t$, this integral can be expressed in terms of the Gamma function,

$$
J(\alpha, t)=\frac{2^{\alpha+1}}{t^{(\alpha+1)}} \mathrm{i}^{(\alpha+1)} \Gamma(\alpha+1)+O(1 / t) .
$$

This result is obtained by deforming the contour of integration onto the upper quarter-circle in the first quadrant and the imaginary axis. The integration along the positive imaginary axis gives the Gamma function.

For $n-1<\operatorname{Re}(\alpha)<n$, where $\mathrm{n}$ is any positive integer, we apply (A 4) and utilize the recurrence relation of the Gamma function $\Gamma(\alpha+1)=\alpha \Gamma(\alpha)$ to obtain

$$
\begin{aligned}
J(\alpha, t) & =\frac{\mathrm{e}^{\mathrm{i} t / 2}}{\mathrm{i}(t / 2)}\left[1+\frac{\mathrm{i}}{t / 2} \alpha+\alpha(\alpha-1)\left(\frac{\mathrm{i}}{t / 2}\right)^{2}+\cdots\right. \\
& +\left(\alpha(\alpha-1) \cdots(\alpha-n+2)\left(\frac{\mathrm{i}}{t / 2}\right)^{n-1}\right]+\frac{2^{\alpha+1}}{t^{\alpha+1}} \mathrm{i}^{(\alpha+1)} \Gamma(\alpha+1)+O\left(\frac{1}{t^{n+1}}\right) .
\end{aligned}
$$

\section{REFERENCES}

Balagondar, P. M., Maslowe, S. A. \& Melkonian S. 1987 The propagation of finite-amplitude waves in a model boundary layer. Stud. Appl. Maths 76, 169-185.

Batchelor, G. K. 1967 An Introduction to Fluid Dynamics. Cambridge University Press.

BELL, G. I. 1990 Interaction between vortices and waves in a simple model of geophysical flow. Phys. Fluids A 2, 575-586.

Bernoff, A. J., Dongen, H. J. H. M. van \& Lichter, S. 1996 The steady boundary layer due to a fast vortex. Phys. Fluids 8, 156-162.

Doligalski, T. L., Smith, C. R. \& Walker, J. D. A. 1994 Vortex interactions with walls. Ann. Rev. Fluid Mech. 26, 573-616. 
DRITSCHEL, D. 1988 a Contour surgery: a topologoical reconnection scheme for extended calculations using contour dynamics. J. Comput. Phys. 77, 240-266.

DritsChel, D. $1988 b$ Contour dynamics and contour surgery: numerical algorithms for extended, high-resolution modelling of vortex dynamics in two-dimensional, inviscid, incompressible flows. J. Fluid Mech. 191, 575-581.

Gradshteyn, I. S. \& Ryzhik, I. M. 1994 Tables of Integrals, Series and Products. Academic Press. Jimenez, J. \& ORLandi, P. 1993 The rollup of a vortex layer near a wall. J. Fluid Mech. 248, 297-313. Milne-Thomson, L. M. 1968 Theoretical Hydrodynamics. Macmillan.

Peridier, V. J., Smith, F. T. \& Walker, J. D. A. 1991 Vortex induced boundary layer separation. Part 1 . The unsteady limit problem $R e \rightarrow \infty$. J. Fluid Mech. 232, 99-131.

Pullin, D. I. 1981 The nonlinear behaviour of a constant vorticity layer at a wall J. Fluid Mech. 108, 401-421.

RAYLEIGH, LORD 1887 On the stability or instability of certain fluid motions, II. In Scientific Papers, vol. 3, pp. 17-23. Cambridge University Press.

Sears, W. R. \& Telionis, D. P. 1975 Boundary-layer separation in unsteady flow. SIAM J. Appl. Maths 28, 215-235.

Stakgold, I. 1967 Boundary Value Problems of Mathematical Physics, Vol II. Macmillan.

Stern, M. E. \& FlierL, G. R. 1987 On the interaction of a vortex with a shear flow. J. Geophys. Res. 92, 10733-10744.

Stern, M. E. \& Pratt, L. J. 1985 Dynamics of vorticity fronts. J. Fluid Mech. 161, 513-532.

Van Dommelen, L. L. \& Shen, S. F. 1980 The spontaneous generation of the singularity in a separating laminar boundary layer. J. Comput. Phys. 38, 125-140.

Walker, J. D. A. 1978 The boundary layer due to rectilinear vortex. Proc. R. Soc. Lond. A 359, $167-188$.

Zabusky, N. J., Hughes, M. H. \& Roberts, K. V. 1979 Contour dynamics for the Euler equations in two dimensions. J. Comput. Phys. 30, 96-106. 